\title{
Searching for old neutron stars with ROSAT
}

\section{Soft X-ray sources in molecular clouds at high galactic latitude}

\author{
R. Danner ${ }^{1,2}$ \\ 1 Max-Planck-Institut für Extraterrestrische Physik, Giessenbachstraße, 85748 Garching, Germany \\ 2 Palomar Observatory, California Institute of Technology, Pasadena, CA 91125, U.S.A.
}

Received December 16, 1996; accepted August 1, 1997

\begin{abstract}
This is the first in a series of three papers aiming to put strong observational constraints on the number of old accreting neutron stars in the Galaxy. Old neutron stars have been predicted to be visible as soft X-ray sources. The brightest sources are expected where the density of the interstellar medium is high. I present an X-ray survey of a complete sample of molecular clouds at high galactic latitudes based on the ROSAT All-Sky Survey. I detect 89 sources inside the projected contours of the molecular clouds and report on a comprehensive identification program. For all sources I searched astronomical catalogs and created finding charts. I also observed all sources at radio frequencies, most of them at $1.4 \mathrm{GHz}$ and at $8 \mathrm{GHz}$. For sources with bright counterparts, optical spectra are presented. For fainter sources, optical CCD images are shown. Of all sources, 54 are securely identified and for the majority of the remaining sources likely identifications are given. I find no candidate for an old accreting neutron star in this sample. From this survey I derive an upper limit to the neutron star density of $50 \mathrm{sr}^{-1}$ at a ROSAT count rate of $0.012 \mathrm{~s}^{-1}$.
\end{abstract}

Key words: surveys - stars: neutron; white dwarfs X-rays: stars — radio continuum: stars

\section{Introduction}

Old isolated neutron stars have sparked the imagination of astronomers since the early days of X-ray astronomy (Ostriker et al. 1970; Helfand et al. 1980). This quest was reignited by the launch of ROSAT and led to several theory papers estimating the detectability of old neutron stars in the ROSAT All-Sky Survey. A general description

\footnotetext{
* Fig. 9 and Fig. 10 are only and Table 2 is additionally available in electronic form at the CDS via http://cdsweb.ustrasbg.fr/Abstract.html
}

of the ROSAT satellite is presented in (Trümper 1983; Pfeffermann et al. 1986) and the All-Sky Survey was described by Voges (1992).

Treves \& Colpi (1991) were the first to calculate in detail the probability of detecting old neutron stars with ROSAT. Later Blaes \& Madau (1993) published a more extensive study. Both groups arrived at the similar conclusion of a high probability to detect old neutron stars. When preliminary results from the Sky-Survey showed that these estimated were too optimistic, both groups reevaluated their calculations (Madau \& Blaes 1994; Colpi et al. 1993) arriving at lower numbers.

A comparison of the pulsar birth rate and the number of known pulsars shows that we have strong indications to believe that the vast majority of neutron stars remains undetected, although more than 650 radio pulsars have been discovered. Only a small number of neutron stars, not active as radio pulsars, are detected as high energy sources. Almost all of which are in close binary systems. Where are the missing old isolated neutron stars?

At birth, a neutron star starts to cool quickly through neutrino emission. Through this process and continuous cooling through photon emission, most of the initial heat is dissipated after less than a million years. Once its surface temperature drops below $10^{5} \mathrm{~K}$, a neutron star is undetectable to most instruments. However, the surface of a neutron star might be heated through accretion from the interstellar medium even after the initial thermal energy content is depleted. The resulting surface temperature depends strongly on the accretion geometry but it is generally expected to be on the order of $10^{6} \mathrm{~K}$. Most estimates have assumed that the emergent surface spectrum is a black-body spectrum. Some theorists have speculated that the surface emission might be harder than a black-body spectrum, see e.g. Zampieri et al. (1995), and have calculated the effect of this assumption on the X-ray background (Zane et al. 1995). However, all calculations confirm that the emission spectrum will not deviate more than 40 percent from a blackbody spectrum. 


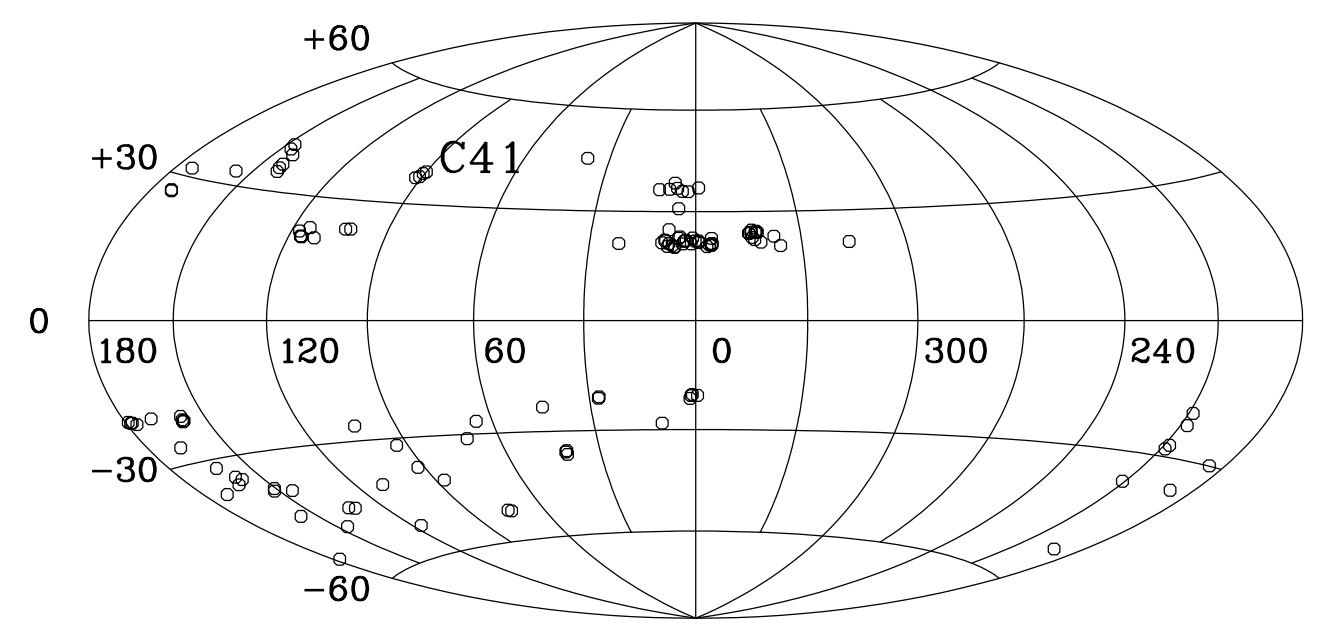

Fig. 1. Distribution of MBM clouds in galactic coordinates. The mark C41 indicates the position of the cloud complex $41-44$ that casts a deep shadow on the diffuse soft X-ray background. An area south of $\delta=-44^{\circ}$ was not covered in this survey because it is unaccessible to the Texas telescope. Circles merely indicate the position of the cloud

Therefore, because of the limited energy resolution available with ROSAT, the spectrum will not be noticeably different from a Planck spectrum. For this reason I will use a simple single-temperature Planck spectrum for all my estimates.

Based on pulsar birth statistics, the total number of neutron stars in our Galaxy is estimated to be $N_{\text {tot }}=$ $10^{8}-10^{9}$. The local neutron star density was derived to be $n \simeq 7.510^{-13} N_{\text {tot }} \mathrm{pc}^{-3}$ with a half density scale height of 280 pc (Madau \& Blaes 1994). A neutron star may be found as close as ten parsecs. Today, only three candidates for old neutron stars accreting from the interstellar medium have been proposed (Stocke et al. 1995; Walter et al. 1996; Haberl et al. 1996). All three candidates will require further work before they are unambiguously identified. A recently published search for old neutron stars (Belloni et al. 1997) has turned up four less well established candidates. Meanwhile, systematic identification of ROSAT sources in the galactic plane (Motch et al. 1997a,b) has sufficiently progressed to demonstrate that the number of candidate objects for old neutron stars is much smaller than expected earlier.

This conflict between the number of old neutron stars predicted and detected motivated me to search the ROSAT All-Sky survey systematically focusing of the regions with the highest expected neutron star densities. The current paper presents the results from a comprehensive identification program of X-ray sources in molecular clouds at high galactic latitude. Paper II covers sources in galactic dark clouds. Here, I present the X-ray source sample and its correlation with the astronomical catalogs SIMBAD and NED. I include finding charts, optical imaging and spectroscopic observations and the results from radio observations of all sources at the VLA. This paper describes the properties of the source sample and the ob- servational data. A later, third, paper will discuss in detail the implications of the surveys in Papers I and II.

\section{Selection and properties of the X-ray sample}

Molecular clouds at high galactic latitudes are the most promising hunting grounds for neutron stars accreting from the interstellar medium because: (1) the density of the interstellar medium (ISM) is high $\left(10-100 \mathrm{~cm}^{-3} \mathrm{com}-\right.$ pared to typically $1 \mathrm{~cm}^{-3}$ or less outside) and we therefore expect higher accretion rates and more luminous sources; (2) the increased column density through the cloud screens out background sources; (3) the location of the cloud at galactic latitudes above $20^{\circ}$ cuts down the number of chance coincidences with unrelated field stars.

An increased absorption due to the higher column density affects all sources and their spectra. For a single source population, distributed isotropically within the sampling volume, I therefore expect fewer and less luminous X-ray sources with on average harder spectra inside the projected boundaries of molecular clouds. Accreting compact objects show the opposite behavior because they are directly interacting with the material in the cloud. Prominent soft X-ray sources without bright optical counterparts are for this reason good candidates for accreting objects inside the clouds. However, high absorption due to material in the vicinity of the source can obscure accreting objects deep inside a cloud. These objects will be lost from the sample in the current search technique. For the time being, I assume that their number is small.

\subsection{The cloud sample}

One sample of clouds is particularly well suited for this search. Magnani, Blitz and Mundy, hereafter MBM, (1985) published a comprehensive survey of the sky 
outside the galactic plane. Areas of apparent optical extinction at latitudes $|b| \geq 20^{\circ}$ and declinations greater than $\delta=-44^{\circ}$ on the Palomar Observatory Sky Survey (POSS) and the White Oak extension were identified by MBM through visual inspection. These candidate areas were then observed at $115 \mathrm{GHz}$ with the $5 \mathrm{~m}$ telescope of the Millimeter Wave Observatory near Fort Davis, Texas because emission at the $\mathrm{CO}(J=1-0)$ transition frequency at $115.2712 \mathrm{GHz}$ is a tracer of molecular clouds. Figure 1 shows the distribution in galactic coordinates of all clouds detected in this survey. The apparent lack of clouds around $b=300^{\circ}$ is due to the declination constraints of the survey.

Fiftyseven clouds in 35 complexes were detected at high galactic latitudes, $|b|>25^{\circ}$. Additionally, 66 clouds were detected at intermediate latitudes $20^{\circ}<|b|<25^{\circ}$. Twentythree of the complexes at high galactic latitudes were later mapped to obtain their morphology. These 23 complexes cover an area of $33.9 \mathrm{deg}^{2}$. Including the clouds that have not been mapped raises the area covered by clouds at high galactic latitudes to $48 \mathrm{deg}^{2}$. The clouds at intermediate latitudes were not mapped by MBM, therefore estimating the covered area in this region is more difficult. An upper limit to the area is the area searched in the ROSAT All-Sky Survey of $0.25 \mathrm{deg}^{2}$ per position. The area searched at intermediate latitudes was $16.5 \mathrm{deg}^{2}$. The total area covered by clouds at high latitudes and at intermediate latitudes is therefore $64.5 \mathrm{deg}^{2}$.

MBM derive a range of densities for the mapped clouds of 35 to $500 \mathrm{~cm}^{-3}$ with a mean value of $140 \mathrm{~cm}^{-3}$. The velocity dispersion of the ensemble is $5.6 \pm 1.2 \mathrm{~km} \mathrm{~s}^{-1}$ and the mean velocity with respect to the local standard of rest is $0.13 \pm 0.11 \mathrm{~km} \mathrm{~s}^{-1}$. The average distance to the clouds is $105 \mathrm{pc}$ and the mean radius of a cloud is $1.7 \mathrm{pc}$.

\subsection{The X-ray sample}

I searched the ROSAT All-Sky Survey for sources that coincide with the cloud sample of MBM. For clouds where no maps were given by the authors, I used a search radius of $0.5^{\circ}$ around the position of the cloud. For the mapped fields, I approximated the often complex shape of the cloud with a mosaic of rectangles. The rectangles were chosen with a generous overlap into empty areas, generally at least $0.5^{\circ}$. All X-ray events detected during the ROSAT All-Sky Survey within the above areas were extracted from the archive. In a second step I binned these event lists into images. On these images, I performed a source detection, following the standard procedures for ROSAT data with some adjustments for survey data. The procedure consisted of an initial source detection, the creation of a background model and a renewed source detection. All sources with smaller separation than the point spread function of the telescope were merged and a likelihood value for the existence of the source at that position was calculated.
During the source detection I chose a reduced likelihood threshold of 8.0, compared to the standard 10.0 value. Accepting a lower value for the likelihood of existence of a source results in an increased number of spurious sources. These are mere fluctuations of the background and are not real objects on the sky. However, the benefit of this is a higher sensitivity to faint sources close to the detection limit of the survey. In this manner, I find 353 sources within $\approx 125 \mathrm{deg}^{2}$, covering an area substantially larger than the area covered by the clouds. I then compared the positions in this initial source list with the maps published by MBM.

I considered any source found inside the lowest contour level of a cloud as a candidate object and included it in the identification program. The lowest contour in the $\mathrm{CO}$ maps of MBM corresponds to an antenna temperature $T_{\mathrm{A}}^{*}$ of $0.5 \mathrm{~K}$. For the unmapped clouds, a maximum distance from the position of the cloud according to MBM of 20 arcminutes was used as a cut-off. I intentionally set the cuts to err on the side of inclusion rather than missing an interesting candidate. Out of the initial 353 sources 90 sources are found inside the projection of an MBM cloud. One source out of this sample was later identified as a double detection leaving me with 89 sources.

The next step was a classification of all sources, based only on their X-ray characteristics. For faint sources the ROSAT Position Sensitive Proportional Counter (PSPC) yields only moderate energy resolution. However, the resolution is sufficient to parameterize the spectrum of a source by a hardness ratio. ROSAT hardness ratios are defined as the number of detected counts in the hard band minus the number of counts in the soft band divided by the total number of counts in the two bands. In this way, any source spectrum is related to a number between -1 for soft sources and +1 for hard sources.

The ROSAT band from 0.1 to $2.0 \mathrm{keV}$ is split into four bands, three of which are independent: $A(0.1-0.4 \mathrm{keV}$ : $11-40), B(0.5-2.0 \mathrm{keV}: 50-200), C(0.5-0.9 \mathrm{keV}$ : $50-200)$ and $D(0.9-2.0 \mathrm{keV}: 90-200)$. The numbers after the colon indicate the selected ROSAT PSPC pulse height channels. The gap between 0.4 and $0.5 \mathrm{keV}$ is due to the carbon absorption edge which renders the PSPC entrance window essentially opaque in this energy range. The hardness ratios HR1 and HR2 are then defined as the ratio of number of detected counts in the respective bands:

$$
\mathrm{HR} 1=\frac{B-A}{B+A} ; \quad \mathrm{HR} 2=\frac{D-C}{D+C}
$$

When a source is not detected in one of the bands a hardness ratio of -1 (for sources only detected in the soft band) or +1 (for sources detected only in the hard band) is assigned. For some faint sources it is only possible to determine HR1 and not HR2, because the source was detected neither in band $\mathrm{C}$ nor $\mathrm{D}$. These sources shown an asterisk in the HR column in Table 2. Two sources are so close to the detection limit that no hardness ratios could 

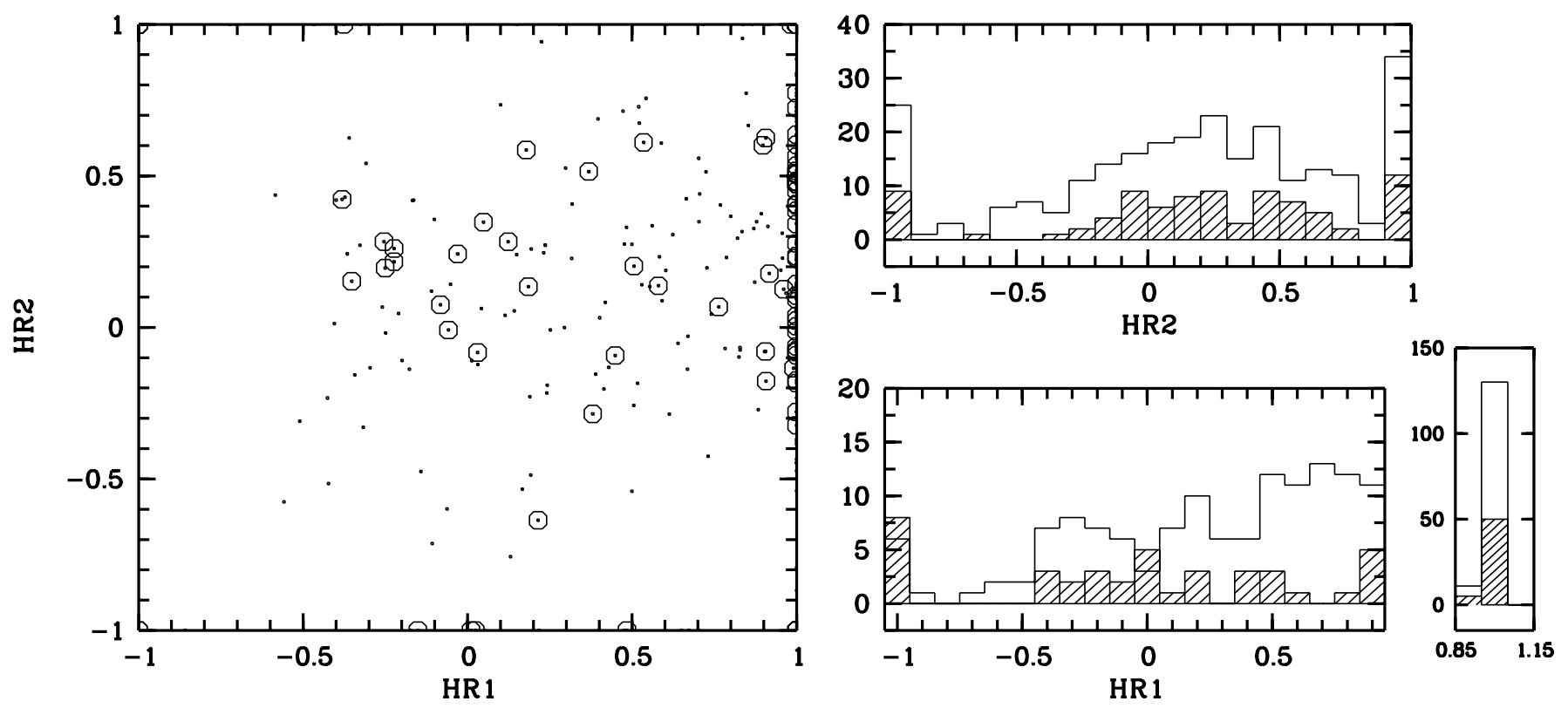

Fig. 2. X-ray Color-Color diagram of the complete sample. left: Single dots — sources not coincident with clouds. Dots surrounded by a circle - sources inside of cloud boundaries. Sources only detected in the soft or the hard band are assigned -1 or +1 values. right: Histogram of source distribution in HR1 (bottom) and HR2 (top). Unhatched histograms - sources outside of clouds. Hatched histograms - source inside of cloud boundaries. (Note: the histograms for HR1 are split into two panels to account for the very high last bin)

be derived. The hardness ratios are generally uncertain in the last digit due to photon statistics.

Figure 2 shows the derived X-ray Color-color diagram, comparing all detected sources with the sources that coincide with MBM clouds. The distribution in the harder band, HR2, appears not to be affected by whether a source is found in front of a cloud or outside the projected boundaries of a cloud. This is not surprising as the column density through the clouds is not sufficient to significantly attenuate X-ray emission above $0.5 \mathrm{keV}$.

In the softer band, HR1, a significant difference between the two groups of sources is found. The ratio of the hardest sources $(\mathrm{HR} 1=+1)$ to the total number of sources is the same for both groups. However, the fraction of soft sources is much higher in the sample coinciding with the clouds than in the sample outside of the clouds.

This finding does not match the expectation from the simple single-population source model. A possible interpretation is a two-population model: a soft, local population and a second hard, more distant population. The harder, preferentially fainter sources, fall below the detection limit of the survey due to the additional absorbing column through a molecular cloud. As a result the distribution is dominated by the soft, local component that does not suffer from absorption because of the proximity to Earth. The same effect is seen with much higher statistical significance in the sample of sources in galactic dark clouds, see Paper II.

\subsection{Modeling the X-ray color-color diagram}

For bright sources it is often possible to fit different source models to their X-ray spectra, to derive a goodness of fit parameter and then decide on the most appropriate model. However, my sources are in general too faint to derive well constrained parameters and I have to assume a source model.

My survey is driven by the idea that the dominant emission from slowly accreting sources will be well described by a black body spectrum. This surface emission has to penetrate intervening material in the immediate vicinity of the source and interstellar material between the source and Earth. These two components can be summarized in a single absorption column to the source. The initial source spectrum plus absorption form the source spectrum arriving at the telescope. This spectrum has to be folded with the energy-dependent vignetting of the imaging system and the detector response matrix.

The initial source spectrum, including absorption, can be written as:

$f(E) \mathrm{d} E \propto \mathrm{e}^{-N_{\mathrm{H}} X(E)} \cdot \frac{2}{c^{2} h^{2}} \cdot \frac{E^{3}}{\mathrm{e}^{E / T}-1} \mathrm{~d} E$

where $N_{\mathrm{H}}$ is the column density in $\mathrm{cm}^{-2}, X(E)$ the photoelectric absorption cross section, $T$ the radiation temperature and $E$ the photon energy. This source model was then folded with the detector response matrix and integrated over the four energy bands. The result is a theoretical 


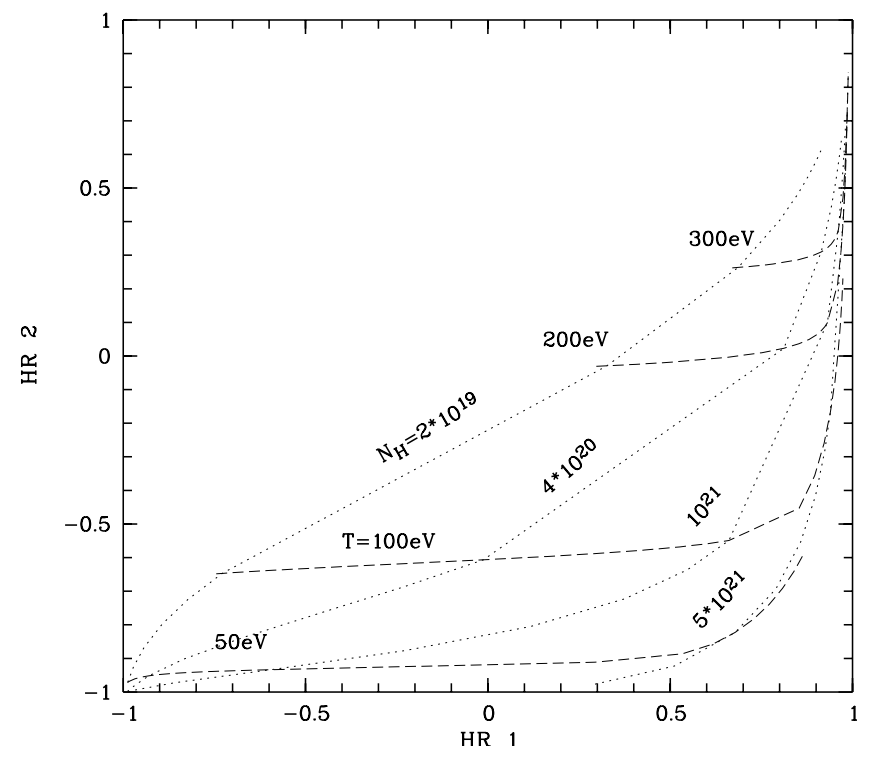

Fig. 3. Assuming a black body source spectrum, the expected hardness ratios are plotted for four given source temperatures (dashed lines) and a range of absorption columns. Dotted lines indicate the ROSAT hardness ratios for a given absorption column and a range of source temperatures

X-ray Color-Color diagram for different source temperatures and absorption columns (see Fig. 3).

At the low end of the temperature range, up to $100 \mathrm{eV}$, even small amounts of absorption will lead to a "hard" value for HR1 because the band between 0.1 and $0.4 \mathrm{keV}$ suffers quickly from even modest absorption. This effect can easily hide intrinsically soft sources in the hard segment of the diagram. Conversely, HR2 responds much more slowly to absorption due to its higher energy. Therefore HR2 is a better parameter in searches for soft sources beyond the local void of the interstellar medium.

\subsection{X-ray to optical flux ratio}

The most powerful classification parameter in the search for compact objects is the ratio of X-ray to optical flux. Previous studies, based on observation made with the EINSTEIN observatory, have pointed out that there is a strong correlation between this ratio and a given class of astronomical objects. Maccararo et al. (1988) order the classes starting with hot B and F stars with $\log \left(f_{\mathrm{X}} / f_{\text {opt }}\right) \approx-4$ through galaxies to AGNs and BL Lac objects with $\log \left(f_{\mathrm{X}} / f_{\text {opt }}\right) \approx+1.5$. They used the following relation to calculate the ratio of $\mathrm{X}$-ray to optical flux (Maccacaro et al. 1988) where the units of the X-ray flux are $\operatorname{erg~s}^{-1} \mathrm{~cm}^{-2}$

$\log \left(f_{\mathrm{X}} / f_{\text {opt }}\right)=\log f_{\mathrm{X}}+\frac{m_{V}}{2.5}+5.37$.

Obtaining CCD photometry on more than 350 sources as an initial screening procedure would require a pro-
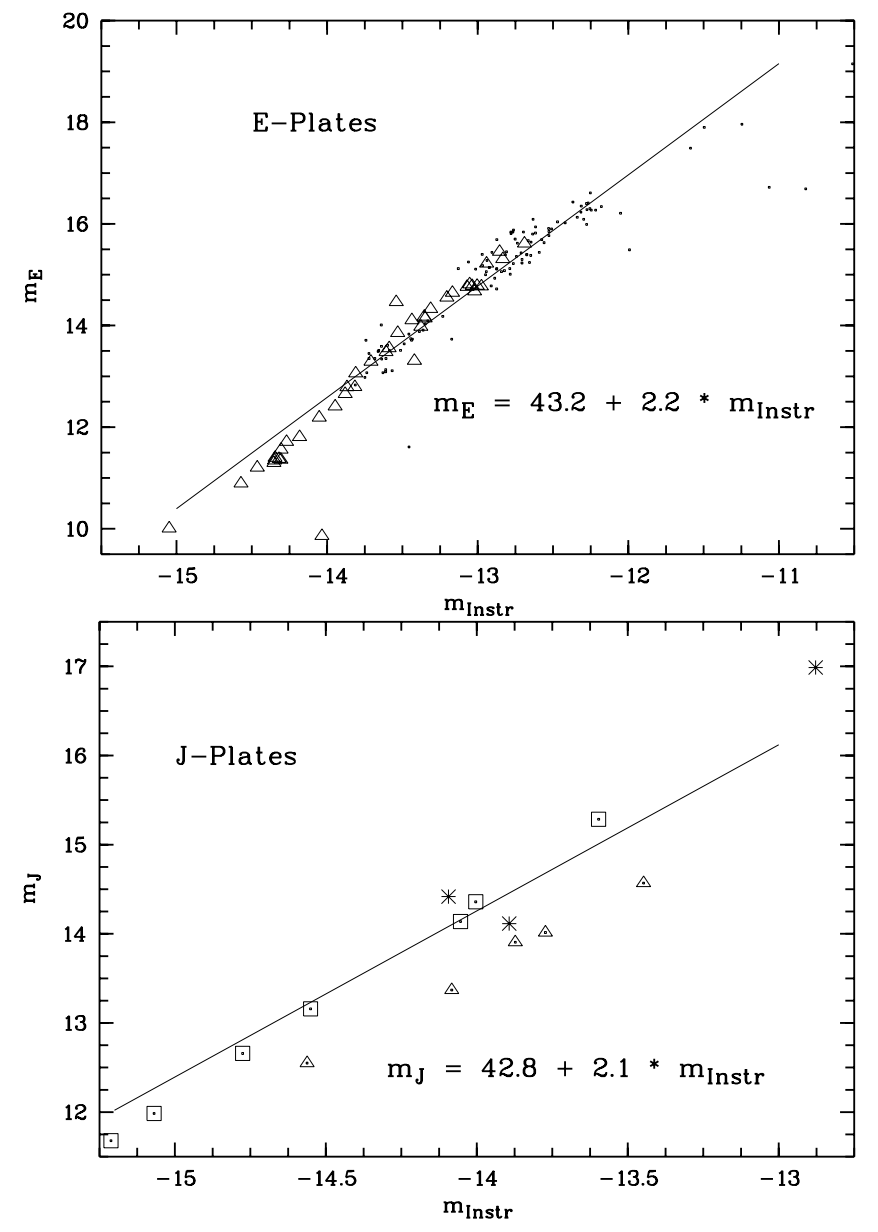

Fig. 4. Magnitude calibration of the Digitized Sky-Survey data sets: top: E-Plates: triangles — Landolt standard stars on same POSS I E-plate; dots - stars from Humphreys et al. from three other POSS I E-plates. A single line is fit to the data for simplicity. bottom: IIIa-J-Plates: Different symbols mark Landolt standard stars from three plates

hibitively large amount of telescope time. Fortunately, digitized versions of the Palomar Observatory Sky Survey (POSS) and the UK-Schmidt plates are available. The CDROM set distributed by the Space Science Telescope Institute supplies data with a spatial resolution of $1.7^{\prime \prime}$. Most of the northern hemisphere data is based on the red Palomar E-plates and the southern hemisphere data is based on the SERC Southern Sky Survey and the SERC $\mathrm{J}$ Equatorial extension.

The data from these CDROMs can be related to standard photometric systems. However, the color systems of the ESO R and SERC J surveys (magnitude $m_{r}, m_{j}$ ) are different from the $B V R$ system $\left(m_{B}, m_{V}, m_{R}\right)$. I use the color transformation from Hörtnagel et al. (1992) and invert their relations to get:

$m_{V}=m_{j}-0.59\left(m_{j}-m_{r}\right)$. 

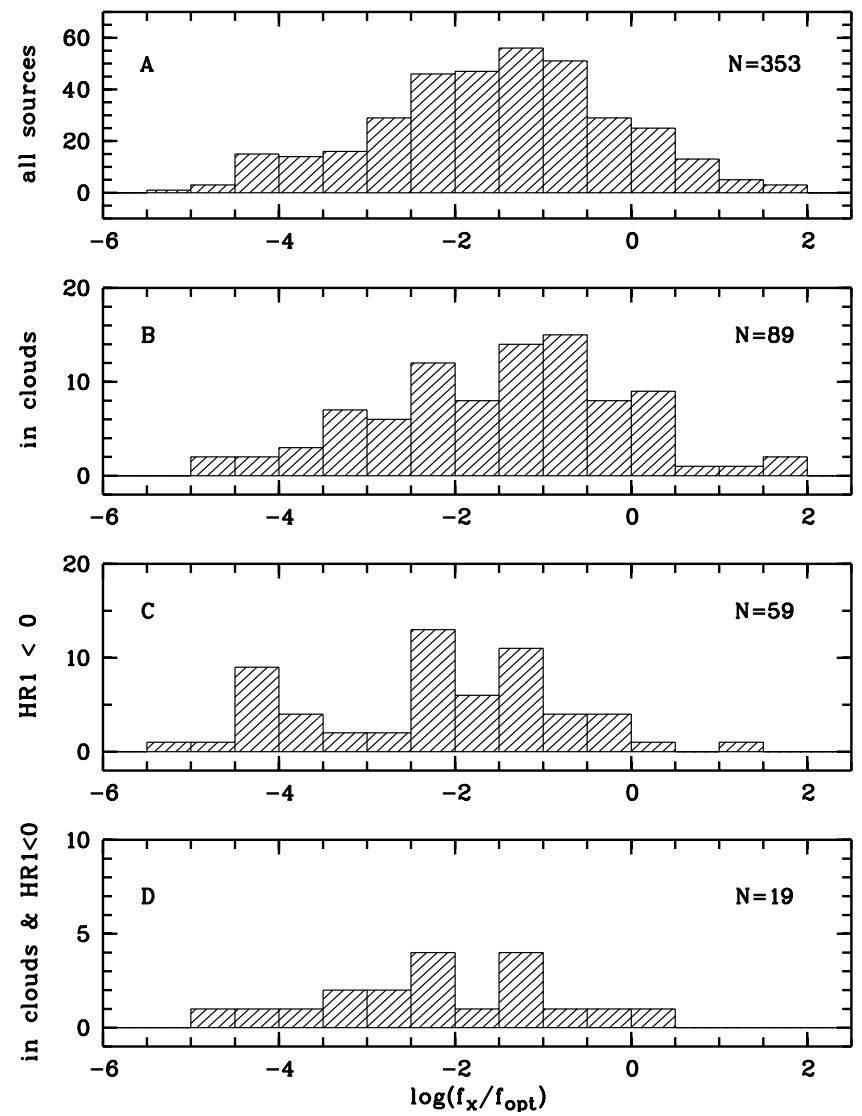

Fig. 5. Distribution of the logarithm of the optical to X-ray flux ratio for different subsamples. Number of sources per logarithm bin: A) all 353 sources; B) only sources inside the projection of an MBM cloud; C) soft sources with a $H R 1<0$; D) distribution of soft sources $(\mathrm{HR} 1<0.0)$ that coincide with clouds

For the Palomar northern plates Humphreys et al. (1991) derive the following color transfer function:

$$
\begin{aligned}
& m_{E}-m_{R}=-0.011+0.148\left(m_{V}-m_{R}\right)+ \\
& +0.058\left(m_{V}-m_{R}\right)^{4} .
\end{aligned}
$$

Ignoring all non-linear terms I simplify this to

$$
m_{E}=m_{R}-0.011+0.148\left(m_{V}-m_{R}\right) .
$$

I looked for a simple method to apply these relationships to the digitized sky survey data set. I used the standard photometry routine magnitude/circle in the MIDAS (Munich Image Data Analysis System) environment to determine "instrumental magnitudes" from the DSS images and applied the above conversions. The result of this approximation for a set of Landolt standards is shown in Fig. 4.

Standard stars on the same survey plate follow closely a linear relation between my derived instrumental magnitudes $\left(m_{\text {Instr }}\right)$ and the magnitudes calculated from the
Johnson $B V R$ system $\left(m_{\mathrm{e}}, m_{\mathrm{J}}\right)$. Because I did not include a correction for different exposure times for each plate, a larger scatter was introduced. This is in particular visible in the plot for the J-Plates. For simplicity I ignore these various offsets, which appear to be of lesser importance for the E-Plates, and fit one regression line for each plate set:

$m_{\mathrm{E}}=43.2+2.2 \cdot m_{\mathrm{Instr}}$

$m_{\mathrm{J}}=42.8+2.1 \cdot m_{\text {Instr }}$.

This rule of thumb serves my purpose of making an initial estimate of the X-ray to optical flux ratio well, but has to be treated very carefully in individual cases. Large color terms can render the estimate meaningless due to the involved systematics. Notwithstanding the above argument, the derived values are an important tool to classify quickly large numbers of sources without any telescope time. For the future, STScI has announced the completion of a calibration set for each individual survey plate that will take individual offsets into account and will yield much more reliable numbers. Currently only one plate of the POSS survey is included in the DSS for each position on the sky and it is therefore not possible to derive color-information from the DSS.

Further assumptions have to be made to estimate the X-ray flux of each source. Most survey sources (with integration times of only $200-300$ s) did not collect enough photons to extract a meaningful spectrum. In the absence of more information on the source spectrum I apply the rough rule for ROSAT: $1 \mathrm{cnt} / \mathrm{sec}=10^{-11} \mathrm{erg} \mathrm{s}^{-1} \mathrm{~cm}^{-2}$ (ROSAT User Handbook, MPE 1997).

As I lack all color information on the optical counterparts, I replace $m_{V}$ with the magnitudes in the photographic pass bands $m_{\mathrm{J}}, m_{\mathrm{E}}$ of the survey plates and obtain:

$\log \left(f_{\mathrm{X}} / f_{\text {opt }}\right)=\log ($ count rate $)+\frac{m_{\mathrm{opt}}}{2.5}-5.63$,

where $m_{\mathrm{opt}}$ is replaced with either $m_{\mathrm{E}}$ or $m_{\mathrm{J}}$, whichever is available.

The distribution of this ratio is plotted for all 353 detected sources in Fig. 5. The four panels in this figure show the distribution of the X-ray to optical flux ratio for all sources (panel A) and three other sub groups. The distribution of this value does not vary significantly for sources found coincident with clouds (panel B), although there might be a hint of fewer sources with high values of $\log \left(f_{\mathrm{X}} / f_{\text {opt }}\right)$. The soft source population, where HR $1<0.0$, has a strong signature of sources with bright optical counterparts and $\log \left(f_{\mathrm{X}} / f_{\text {opt }}\right)$ between -4.5 and -3.5 . Finally, the soft sources, HR $1<0.0$ that coincide with clouds appear to be completely devoid of sources with high values for this ratio. 

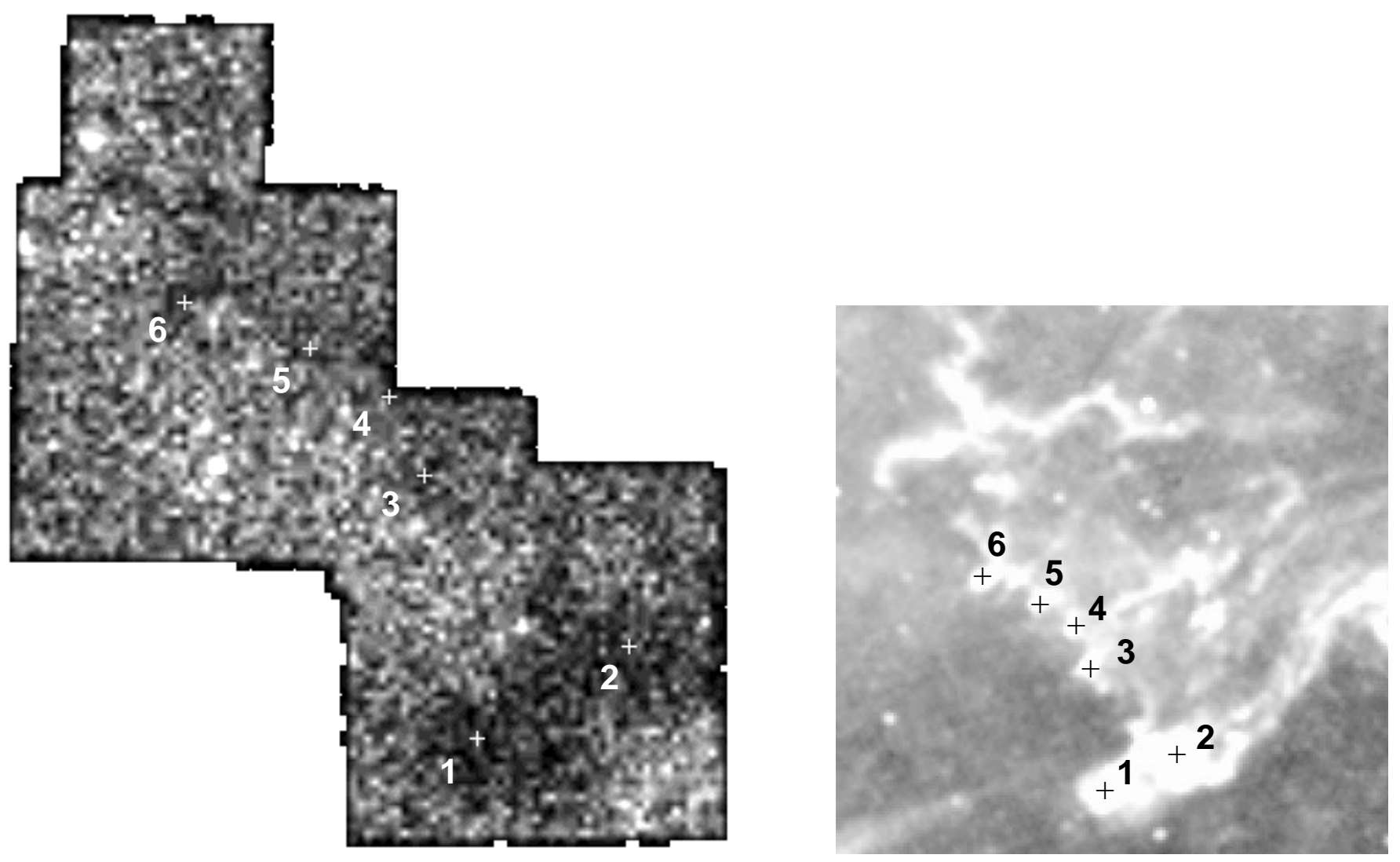

Fig. 6. ROSAT All-Sky Survey image of MBM clouds 41 to 44 (left) and IRAS $100 \mu \mathrm{m}$ image of the same region (right). Numbered crosses mark corresponding locations on the sky. In both images lighter shades indicate increased emission. The shadow on the diffuse X-ray background (left) closely matches the bright emission band in the infrared image (right). The images extend over approximately 3 by 3 degrees, north is up and East to the left

\subsection{Diffuse structures}

In the search for point sources I also discovered a previously undescribed extended feature. The MBM clouds 41 through 44 cast a distinct shadow on the soft X-ray background. The left panel in Fig. 6 shows the ROSAT image in the 0.1 to $0.4 \mathrm{keV}$ range. The right panel shows an image from the IRAS $100 \mu \mathrm{m}$ survey of the same area. Numbered crosses mark corresponding coordinates in the $\mathrm{X}$-ray and the IRAS image. A lighter shade corresponds in both images to a higher intensity. The intensity of areas with reduced diffuse X-ray emission and outside differs by up to a factor of 2.5. The structure of the diffuse background as seen with ROSAT has been most recently described by Snowden et al. (1995). Shadowing structures have been described on several occasions in the past (e.g. Snowden et al. 1993). However, the complex of clouds 41 to 44 casts one of the cleanest and deepest shadows so far identified. The absorption contours in the X-ray image accurately trace the areas of emission on the IRAS image. The IRAS image shows that the apparently isolated peaks of CO emission (Magnani et al. 1985) belong in fact to the same structure.

\section{Correlation with existing data bases}

\subsection{Uncertainty in the X-ray position}

The effective point spread function of the ROSAT telescope is significantly broader during the All-Sky-Survey than during the pointed observations. This is due to the scanning mode where the PSF is averaged over a wide range of off-axis angles. For most of my sources the number of detected photons is too low to recover this resolution. For consistency I choose a source independent 90 percent error radius $r_{90}$ of 30 arcsec, regularly used in ROSAT source identification (Hasinger priv. com.). Motch et al. (1997a) calculate an upper limit of $r_{90}=32$ arcsec for their extensive identification program and find that this is a conservative limit. The result of my identification program supports this finding, see Fig. 7.

\subsection{Catalog search}

I searched the astronomical catalogs provided by SIMBAD and the NASA Extra Galactic Database (NED) for objects at the X-ray position. In SIMBAD a search radius of 
1 arcminute and in NED a search radius of 5 arcminutes was used. Any object within 30 arcsecond of the reported $\mathrm{X}$-ray position was considered a counterpart to the source as long as there was no compelling evidence found to contradict this. Of the 89 sources coinciding with clouds, 11 were readily identified with bright $\left(m_{V}<10.6\right)$ stars and 6 others with nearby low mass dwarf stars and one emission line star $\left(m_{V}, 14.6\right)$. Two sources coincide with known quasi-stellar objects (QSOs) and three others with known radio sources. The Abell cluster 0496 was found only 1.5 arcminutes distant from RX J0434.5-1334. Table 2 shows the names of all identified SIMBAD sources.

\subsection{Finding charts}

In addition to the DSS images, I created finding charts from data in the Automated Plate Measuring machine catalog. The APM group at Cambridge independently scanned plates from the Palomar Observatory Sky Survey. The significant advantage of the APM catalog over the DSS scans is the additional color information as both red $(\mathrm{E})$ and blue $(\mathrm{J})$ plates have been scanned. The detection limits are around $21.5 \mathrm{mag}$ in $\mathrm{J}$ and $20 \mathrm{mag}$ in E. The APM data base is an object list, including an automated classification into stellar and non-stellar objects, rather than a collection of images. This can cause confusion when large extended structures such as bright emitting clouds are encountered. A comparison between the images from the DSS and the APM scans can then resolve ambiguities in the morphology of the source. I give finding charts for all objects of which spectra have been taken in Fig. 9 .

\section{Optical and radio identification program}

The majority of sources required further work beyond the catalog searches, either because the objects were too faint or because more information on their optical spectra was required to resolve ambiguities.

\subsection{Radio maps}

I observed all X-ray source positions in a program of short exposures at the Very Large Array Radio Telescope (VLA) in New Mexico. Between 9 September 1995 and 25 October 1995 a total of 20.5 hours were spent in two bands, at $1.425 \mathrm{GHz}$ ( $L$-band) and $8.439 \mathrm{GHz}$ ( $X$-band). I included all source positions in the $L$-band program and about half of the sources in the $X$-band observations. Some observations were lost due to technical problems at the telescope and some due to radio interference. In a few cases the detection limit at the source position was strongly limited by side lobes from strong sources outside the primary beam. All observations prior to October 1995 were done in the hybrid AnB configuration resulting in an elliptical beam that varies with the elevation of the object. The remaining observations were conducted in the more extended B configuration, with a more circular beam.
Table 1 summarizes all fields with detected radio sources detected close enough to the X-ray position to serve as plausible radio counterparts. Table 2 shows in Col. B if a source position has a successfully reduced radio map associated with it. A successful radio map is one in which the observation is free from major radio interference, was successfully calibrated and cleaned to an acceptable level. The typical root mean square noise level is between 250 and $350 \mu \mathrm{Jy}$ at $L$-band and 150 to $200 \mu \mathrm{Jy}$ at $X$-band. These values are a factor 2-3 higher than the expected theoretical thermal noise. The reasons for the high noise levels are mostly large zenith angles, the increased background in areas close to the galactic plane and confusing sources. Cleaning the images beyond the current noise level proved to be prohibitively expensive in terms of time and was not justified by the objective to find strong isolated sources. All VLA data were reduced and calibrated within the AIPS software packet distributed by the National Radio Astronomy Observatory.

\subsection{Optical observations}

I took optical spectra and CCD images of most sources during 15 observing nights at Mt. Palomar between 28 June 1995 and 27 December 1995. For low resolution spectra of stars brighter than $m_{V}=16$ I used the Echelle spectrograph in the long slit mode at the Palomar 60-inch telescope. For spectra of fainter objects, down to $m_{V} \approx 18$, I used primarily the Double Spectrograph at the Cassegrain focus of the 200-inch Hale telescope. For some fields I also used the COSMIC prime focus instrument at the Hale telescope. The optical data were reduced using standard routines in the IRAF software suite.

\section{Identification and statistics}

My identification strategy was to search the X-ray error circle for a counterpart until a plausible counterpart was found. Plausible means an object with either a characteristic signature (color, spectrum) for a counterpart to an X-ray source or where the X-ray to optical flux ratio was within the typical ratio for a given source type. AGN spectra, broad Balmer lines and forbidden [OIII] emission were always taken as a strong indicator for the X-ray counterpart, regardless of the presence of radio emission. $\mathrm{M}$ dwarf spectra with or without $\mathrm{H}_{\alpha}$ emission were also strong counterpart candidates. In a few cases bright stars are found just outside the error circle. These are quite likely the counterparts to the X-ray sources, as other identification programs have shown.

The analysis of the X-ray data and the multiwavelength follow-up program led to the identification of the majority of sources in my sample. Out of 89 sources 54 have firm identifications; for the remaining 34 sources the current status of the identification process is presented. More spectroscopic data would be required to resolve the 
Table 1. All detected VLA radio sources. Internal identification number, X-ray position from ROSAT Sky Survey, radio position ( $3.6 \mathrm{~cm}$ emission peak if source was detected, else peak of $20 \mathrm{~cm}$ emission), peak flux per beam and RMS noise in mJy at $20 \mathrm{~cm}$ and $3.6 \mathrm{~cm}$, distance from X-ray position in arcseconds, in this column "I" indicates that this source is associated with the X-ray source. Asterisk in peak level: no source. Asterisks in peak and noise columns: no observation

\begin{tabular}{|c|c|c|c|c|c|c|c|c|c|c|}
\hline & \multicolumn{2}{|c|}{ X-ray Position } & \multicolumn{2}{|c|}{ Radio Position } & \multicolumn{2}{|c|}{$20 \mathrm{~cm}$} & \multicolumn{2}{|c|}{$3.6 \mathrm{~cm}$} & & \\
\hline$\overline{\mathrm{ID}}$ & $\alpha_{(2000)}$ & $\delta_{(2000)}$ & $\alpha_{(2000)}$ & $\delta_{(2000)}$ & Peak & Noise & Peak & Noise & Dist. & \\
\hline 30 & $00: 10: 29.28$ & $+20: 47: 38.5$ & $00: 10: 28.781$ & $+20: 47: 49.0$ & 49.9 & 0.81 & * & * & 12.8 & $\mathrm{I}$ \\
\hline 69 & $03: 20: 38.02$ & $+11: 24: 55.0$ & 03:20:38.02 & $+11: 24: 51.5$ & 8.8 & 0.44 & * & * & 3.5 & I \\
\hline 76 & 04:02:42.00 & $+01: 59: 54.4$ & 04:02:48.18 & $+01: 57: 58.9$ & 8.6 & 0.80 & $*$ & * & 148.0 & \\
\hline 75 & 04:03:14.15 & $+02: 15: 21.4$ & 04:03:09.36 & $+02: 11: 32.1$ & 141.0 & 1.4 & * & * & 240.3 & \\
\hline 34 & $04: 22: 02.65$ & $+19: 32: 20.8$ & $04: 21: 59.55$ & $+19: 32: 06.8$ & 2.5 & 0.24 & * & * & 48.0 & I \\
\hline 37 & $04: 22: 08.36$ & $+19: 15: 17.3$ & 04:22:02.65 & $+19: 32: 27.8$ & 7.5 & 0.29 & * & * & 1034.0 & \\
\hline 48 & 04:30:04.28 & $+18: 13: 42.7$ & 04:30:04.52 & $+18: 16: 25.4$ & * & * & 21.4 & 0.60 & 162.7 & \\
\hline 08 & 09:29:49.17 & $+65: 22: 53.0$ & 09:29:49.17 & $+65: 22: 53.0$ & 17.6 & 1.4 & $*$ & 0.19 & 0.0 & I \\
\hline 07 & $09: 37: 27.21$ & $+65: 38: 28.4$ & 09:37:28.43 & $+65: 38: 31.2$ & 12.4 & 0.44 & * & * & 15.6 & \\
\hline 84 & $15: 37: 06.46$ & $-24: 47: 35.4$ & $15: 37: 11.47$ & $-24: 50: 19.8$ & 9.48 & 0.70 & * & 0.21 & 180.0 & \\
\hline 17 & $16: 45: 47.79$ & $+59: 57: 36.9$ & $16: 45: 31.92$ & $+59: 59: 25.3$ & 5.93 & 0.37 & * & 0678 & 233.0 & \\
\hline 14 & $16: 58: 14.45$ & $+60: 56: 15.7$ & $16: 58: 28.36$ & $+60: 54: 39.4$ & 16.5 & 0.38 & * & 0.16 & 204.0 & \\
\hline 12 & $16: 58: 32.42$ & $+61: 59: 46.3$ & $16: 58: 35.70$ & $+61: 59: 35.8$ & 3.9 & 0.28 & 2.39 & 0.17 & 43.5 & I \\
\hline 88 & $19: 19: 31.29$ & $-36: 39: 25.6$ & 19:19:38.71 & $-36: 42: 53.8$ & 67.5 & 1.9 & * & 0.19 & 233.5 & \\
\hline 25 & 23:00:03.44 & $+24: 44: 51.3$ & 23:00:04.17 & $+24: 44: 46.7$ & 64.3 & 1.0 & 7.05 & 0.19 & 11.6 & I \\
\hline 27 & 23:02:10.86 & $+24: 17: 35.4$ & $23: 02: 10.52$ & $+24: 17: 21.2$ & 3.3 & 0.15 & $*$ & * & 15.0 & I \\
\hline 57 & 23:04:51.90 & $+14: 12: 41.4$ & 23:05:05.02 & $+14: 14: 24.6$ & 24.1 & 0.61 & * & 0.13 & 220.9 & \\
\hline 56 & 23:05:58.54 & $+14: 13: 25.0$ & 23:05:58.21 & $+14: 13: 10.6$ & 1.2 & 0.15 & * & 0.37 & 15.2 & I \\
\hline 39 & $23: 07: 47.11$ & $+19: 01: 17.1$ & $23: 07: 45.53$ & $+19: 01: 20.3$ & 328 & 5.3 & * & * & 23.6 & I \\
\hline 35 & 23:08:12.31 & $+19: 30: 09.9$ & $23: 08: 16.27$ & $+19: 31: 04.1$ & 18.2 & 0.54 & * & * & 79.8 & \\
\hline 47 & 23:09:01.47 & $+18: 20: 21.2$ & 23:09:02.69 & $+18: 19: 50.0$ & 3.0 & 0.14 & * & * & 36.1 & I \\
\hline 50 & $23: 12: 31.24$ & $+18: 06: 22.4$ & $23: 12: 32.95$ & $+18: 03: 15.1$ & 10.5 & 0.56 & * & 0.15 & 189.0 & \\
\hline
\end{tabular}

ambiguous cases with several optical counterparts inside the error box. However, in most cases the most likely counterpart has already been identified. Table 2 summarizes the results of this program. Previously known objects are listed with their names. In many cases optical spectra were required to resolve ambiguities, see Fig. 8. I created finding charts for all objects that I took spectra of, see Fig. 9. Arrows on the finding charts mark the objects. These finding charts are based on the APM scans of the POSS I plates. CCD frames are reproduced for all fields where no good APM or DSS finding chart was available, Fig. 10. The APM finding charts, the CCD frames and the identification spectra are all referenced to Table 2 through their internal ID number which is easier to handle than the full $\mathrm{X}$-ray position. Table 3 finally lists remarks on the rationale for each individual identification.

Figure 7 presents the distribution of the separation between the X-ray source and the identified counterpart for my sample. The distribution is shown first as the number of identifications as a function of the separation. A second panel shows the same distribution but normalized by the radius. In this normalization the increasing sampling area for each radius bin has been removed from the distribution. The distribution confirms my initial assumption that most sources will have counterparts within 30 arc- seconds of the X-ray source position. In a few cases very bright stars that burn out the point spread function are nominally found at larger distances.

Of the 54 firm identifications which are cataloged objects or objects with optical identification spectra, 34 are stars and 20 are extragalactic objects. Of the remaining sources, all but two have several counterparts in their error box. A few of the sources at the faint flux limit are expected to be spurious detections due to the lower likelihood threshold that I used in the source detection process. No unidentified source shows the X-ray characteristics that I expect from a soft thermal source nor is any of them luminous enough to indicate an unusual X-ray to optical flux ratio in the presence of the counterparts inside the error box. The only soft and bright source in the entire 89 source sample, 49, stands out in its X-ray characteristics and was readily identified with a DC white dwarf star (see identification spectrum). A considerably larger amount of telescope time would have to be invested to resolve all ambiguities at the end of which I expect that most of the suggested candidates would be confirmed.

\section{Summary}

A detailed discussion of this survey, together with the results from Paper II, will be published as a later 

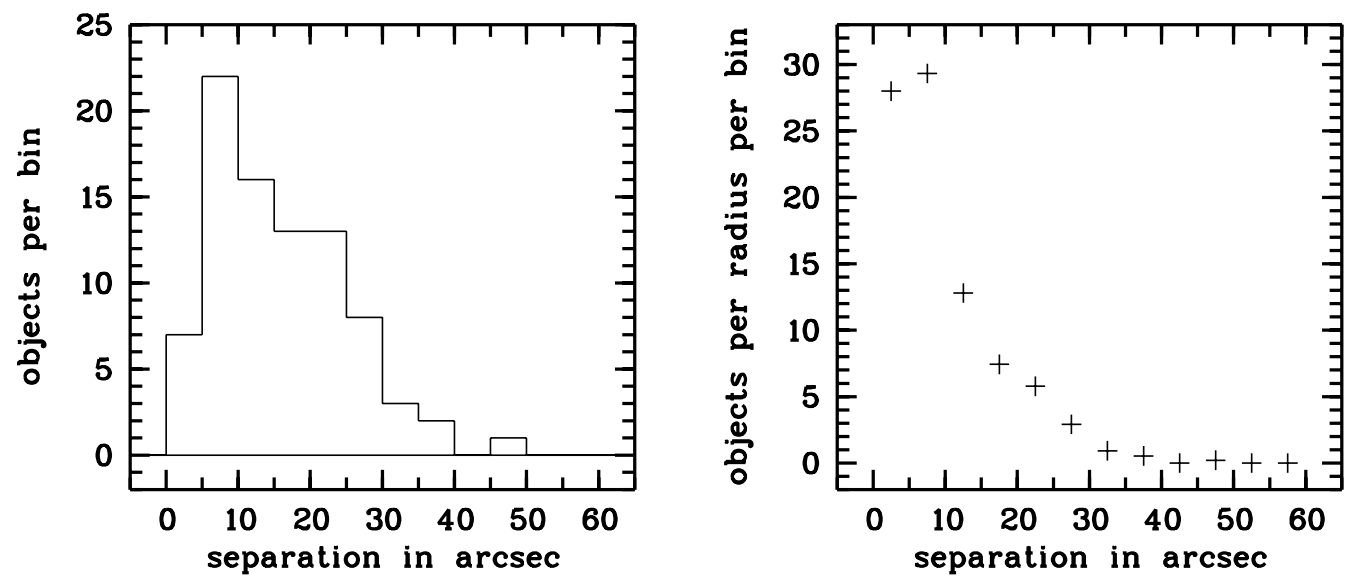

Fig. 7. Separation between the X-ray sources and their counterpart. left: number of counterparts found in a given radius interval. Distance in arcsec. right: same distribution but normalized by the radius

paper. Here, I will only briefly summarize the results of the current survey. I found one white dwarf, several late type stars, $\mathrm{M}$ stars with and without emission lines and a large population of AGNs. I identified no candidate for an accreting neutron star over the sample area of $64.5 \mathrm{deg}^{2}$. Because of the reduced detection threshold for X-ray sources I expect to be complete to the 99 percent level at a count rate of $0.012 \mathrm{~s}^{-1}$. This implies that at most one of the 89 sources might have not been detected in the X-ray survey. An upper limit of one source in the $64.5 \mathrm{deg}^{2}$ survey area translates to $\approx 50$ such sources per steradian $\left(50 \mathrm{sr}^{-1}\right)$. This upper limit to the number of old accreting neutron stars, assuming $10^{8}$ neutron stars, is a factor of 1.6 lower than the lowest value of the prediction for isotropic accretion, and more than a factor of 35 lower than we expect for polar-cap accretion (Blaes \& Madau 1993).

Acknowledgements. This article is based on research conducted during my three year visit to the California Institute of Technology. I want to thank Prof. S.R. Kulkarni for his hospitality, support and countless discussions during this time. I am indebted to J. Trümper for his support of this project and many fruitful suggestions. The ROSAT project is supported by the German Bundesministerium für Bildung und Wissenschaft (BMBW/DARA) and the Max-Planck-Society. This research has made use of the NASA/IPAC extragalactic database NED) which is operated by the Jet Propulsion Laboratory, Caltech, under contract with the National Aeronautics and Space Administration, and of the SIMBAD database, operated at CDS, Strasbourg, France. The Digitized Sky Survey (DSS) was produced at the Space Telescope Science Institute under U.S. Government grant NAG W-2166. The National Radio Astronomy Observatory (NRAO) is operated by Associated Universities, Incorporated, under cooperative agreement with the National Science Foundation.

\section{References}

Belloni T., Zampieri L., Campana S., 1997, A\&A 319, 525-534 Blaes O., Madau P., 1993, ApJ 403, 690

Colpi M., Campana S., Treves A., 1993, A\&A 278, 161

Haberl F., Pietsch W., Motch C., Buckley D.A.H., IAU Circular 6445, 6th August 1996

Helfand D.J., Chanan G.A., Novick R., 1980, Nat 283, 337-343

Hörtnagel A.M., Kimeswemger S., Weinberger R., 1992, A\&A 262,369

Humphreys R.M., Landau R., Ghigo F.D., Zumbach W., 1991, AJ 102, 395

Madau P., Blaes O., 1994, ApJ 423, 748

Maccacaro T., Gioia I.M., Wolter A., Zamorani G., Stocke J.T., 1988, ApJ 326, 680

Magnani L., Blitz L., Mundy L., 1985, ApJ 295, 402

Motch C., Guillout P., Haberl F., Pakull M., Pietsch W., Reinsch K., 1997a, A\&A 318, 111-133

Motch C., Guillout P., Haberl F., Pakull M.W., Pietsch W., Reinsch K., 1997b, A\&AS 122, 201-213

Ostriker J.P., Rees M.J., Silk J., 1970, Astrophys. Lett. 6, 179

Pfeffermann E., Briel U.G., Hippmann H., Kettenring G., Metzner G., Predehl P., Rger G., Stephan K.H., Zombeck M.V., Chappel J., Murray S.S., 1986, SPIE 733, 519

Snowden S.L., McCammon D., Verter F., 1993, ApJ 409, L21

Snowden S.L., Freyberg M.J., Plucinsky P.P., Schmitt J.H.M.M., Trümper J., Voges W., Edgar R.J., McCammon D., Sanders W.T., 1995, ApJ 454, 643

Stocke J.T., Wang Q.D., Perlman E.S., Donahue M., Schachter J., 1995, AJ 109, 1199

Treves A., Colpi M., 1991, A\&A 241, 107

Trümper J., 1983, Adv. Space Res. 2, 241

Voges W., 1992, in Proceeding of Satellite Symposium 3, "International Space Year Conference", ESA ISY-3, p. 9

Walter F.M., Wolk S.J., Neuhäuser R., 1996, Nat 379, 233

Zampieri L., Turolla R., Zane S., Treves A., 1995, ApJ 439, 849-853

Zane S., Turolla R., Zampieri L., Colpi M., Treves A., 1995, ApJ 451, 739-746

Zimmermann H.U., et al., 1994, MPE Report 257 
Table 2. Summary of the identification program of all 89 sources found within the lowest contours of the MBM cloud maps. Columns are: ID: Internal reference number; X-ray source position in equinox 2000.0; Rate: source count rate in cts $\mathrm{s}^{-1}$; Error: error in count rate in $\operatorname{cts~s}^{-1}$; HR1, HR2: hardness ratios $\left(^{*}\right.$ : source was not detected when data was split into two spectral channels); Name: catalog name of object; Type: Spectral Type; $m$ : visual magnitude of counterpart; O-E: color index from APM scans; dist.: separation between X-ray source and proposed counterpart; B: bands in which the source has been observed at the VLA; log: logarithm of X-ray to optical flux ratio.

$\dagger$ not detected in snapshot; $\ddagger$ in visual binary with M4V. $(*)$ : new Identification; $(+)$ new classification

\begin{tabular}{|c|c|c|c|c|c|c|c|c|c|c|c|c|c|}
\hline \multirow[b]{2}{*}{ ID } & \multicolumn{6}{|c|}{ X-ray Source Parameters } & \multicolumn{2}{|c|}{ Identification } & \multicolumn{3}{|c|}{ POSS } & \\
\hline & $\alpha_{(2000)}$ & $\delta_{(2000)}$ & Rate & Error & HR1 & HR2 & Name & Type & $m$ & $\mathrm{O}-\mathrm{E}$ & dist & $\mathrm{B}$ & $\log$ \\
\hline 30 & 00:10:29.3 & $+20: 47: 38$ & 0.035 & 0.013 & 1.00 & 0.28 & $87 \mathrm{~GB}$ & & 17.86 & 0.56 & 12.8 & LX & 0.1 \\
\hline 52 & $01: 15: 20.3$ & $+16: 48: 19$ & 0.017 & 0.007 & 1.00 & 1.00 & new ID & K0III & 12.91 & 0.96 & 38.8 & LX & -2.2 \\
\hline 40 & 02:25:05.4 & $+18: 46: 55$ & 0.177 & 0.029 & 1.00 & 0.50 & $87 \mathrm{~GB}$ & & 18.67 & 1.41 & 12.2 & & 1.1 \\
\hline 74 & $03: 17: 46.2$ & $+10: 17: 35$ & 0.011 & 0.006 & 1.00 & 0.34 & & & 17.71 & 1.68 & 23.6 & $\mathrm{~L}$ & -0.5 \\
\hline 69 & 03:20:38.0 & $+11: 24: 55$ & 0.055 & 0.011 & 1.00 & 0.72 & $\operatorname{RadioS}(*)$ & & 19.41 & $>1.15$ & 3.5 & $\mathrm{~L}$ & 0.5 \\
\hline 63 & $03: 22: 02.5$ & $+12: 28: 45$ & 0.015 & 0.007 & -1.00 & $*$ & & & O20.37 & $<0.37$ & 27.9 & $\mathrm{~L}$ & 0.7 \\
\hline 73 & $03: 22: 44.3$ & $+10: 37: 27$ & 0.015 & 0.006 & 0.02 & -1.00 & $\operatorname{SIMBAD}(+)$ & $\mathrm{M} 4 \mathrm{~V}$ & V14.6 & & 10.2 & $\mathrm{~L}$ & -1.6 \\
\hline 64 & 03:23:04.0 & $+12: 24: 40$ & 0.019 & 0.008 & -1.00 & $*$ & & & O20.17 & $<0.17$ & 22.2 & $\mathrm{~L}$ & 0.7 \\
\hline 66 & $03: 23: 22.2$ & $+11: 41: 16$ & 0.123 & 0.018 & -0.22 & 0.26 & $\operatorname{SIMBAD}(+)$ & $\mathrm{M} 4 \mathrm{~V}$ & $\mathrm{~V} 12.2$ & & 16.6 & $\mathrm{~L}$ & -2.5 \\
\hline 71 & $03: 23: 30.6$ & $+10: 48: 00$ & 0.014 & 0.006 & 1.00 & -0.32 & $(*)$ & K3 & 13.9 & 2.13 & 6.2 & $\mathrm{~L}$ & -1.9 \\
\hline 62 & $03: 24: 10.0$ & $+12: 37: 41$ & 0.054 & 0.012 & 0.91 & -0.08 & SAO 93416 & K0III-IV & V 6.0 & & 1.2 & $\mathrm{~L}$ & -4.5 \\
\hline 61 & 03:24:38.4 & $+12: 53: 39$ & 0.017 & 0.007 & 1.00 & 0.48 & & & 18.17 & 1.15 & 1.7 & $\mathrm{~L}$ & -0.1 \\
\hline 68 & $03: 25: 25.2$ & $+11: 27: 53$ & 0.013 & 0.006 & 1.00 & 0.64 & & & 17.52 & 1.88 & 15.0 & $\mathrm{~L}$ & -0.5 \\
\hline 72 & $03: 25: 48.2$ & $+10: 47: 02$ & 0.024 & 0.008 & 1.00 & 0.52 & RadioS $(*)$ & RadioS & 17.85 & 2.08 & 26.0 & $\mathrm{~L}$ & -0.1 \\
\hline 67 & $03: 26: 20.8$ & $+11: 31: 00$ & 0.101 & 0.018 & -0.06 & -0.01 & $(*)$ & $\mathrm{M} 4 \mathrm{~V}$ & 14.66 & 1.56 & 9.7 & $\mathrm{~L}$ & -0.8 \\
\hline 65 & 03:26:46.1 & $+12: 10: 11$ & 0.015 & 0.007 & 0.91 & 0.62 & $z=0.21(*)$ & AGN & 18.88 & 1.63 & 11.4 & $\mathrm{~L}$ & 0.1 \\
\hline 70 & 03:28:02.2 & $+11: 14: 34$ & 0.065 & 0.016 & -0.35 & 0.15 & binary $(*)$ & M4V:e & 15.07 & 2.34 & 11.0 & $\mathrm{~L}$ & -0.8 \\
\hline 22 & $03: 28: 58.7$ & $+31: 14: 18$ & 0.043 & 0.011 & 1.00 & 0.02 & $\mathrm{BD}+30547$ & K0 & V10.3 & & 19.9 & $\mathrm{~L}$ & -2.9 \\
\hline 21 & $03: 29: 14.3$ & $+31: 22: 47$ & 0.018 & 0.008 & 1.00 & 0.60 & & & 18.40 & $>2.99$ & 16.1 & $\mathrm{~L}$ & 0.0 \\
\hline 77 & 04:01:10.9 & $+00: 04: 11$ & 0.022 & 0.010 & 1.00 & 0.39 & $z=0.47(*)$ & AGN & 17.71 & 0.65 & 13.0 & $\mathrm{~L}$ & -0.2 \\
\hline 76 & 04:02:42.0 & $+01: 59: 54$ & 0.032 & 0.011 & 1.00 & 0.48 & $z=0.14\left(^{*}\right)$ & $\mathrm{AGN}$ & 16.83 & 1.70 & 12.7 & $\mathrm{~L}$ & -0.4 \\
\hline 75 & 04:03:14.2 & $+02: 15: 21$ & 0.013 & 0.007 & 1.00 & 1.00 & & & 18.09 & $>3.32$ & 24.2 & $\mathrm{~L}$ & -0.3 \\
\hline 33 & $04: 21: 45.7$ & $+19: 34: 01$ & 0.017 & 0.007 & 1.00 & 0.04 & $(*)$ & $\mathrm{M} 1.5 \mathrm{~V}: \mathrm{e}$ & 14.63 & 3.98 & 37.1 & $\mathrm{~L}$ & -1.5 \\
\hline 34 & 04:22:02.6 & $+19: 32: 20$ & 0.037 & 0.010 & 1.00 & 0.47 & T-Tauri & & & & 48.0 & & $>1.3$ \\
\hline 37 & 04:22:08.4 & $+19: 15: 17$ & 0.116 & 0.019 & -0.25 & 0.20 & EinsteinS & $\mathrm{M} 4.5 \mathrm{~V}: \mathrm{e}$ & V12.9 & & 6.3 & $\mathrm{~L}$ & -1.4 \\
\hline 31 & 04:22:18.3 & $+19: 50: 47$ & 0.134 & 0.019 & 1.00 & 0.51 & ${\mathrm{MS}-\mathrm{Src}^{\dagger}}^{\dagger}$ & QSO & & & 18.8 & $\mathrm{~L}$ & \\
\hline 32 & $04: 23: 23.1$ & $+19: 39: 40$ & 0.019 & 0.008 & 0.01 & -1.00 & $\mathrm{BD}+19708$ & $\mathrm{~K} 2 \mathrm{~V}$ & V 9.4 & & 11.3 & $\mathrm{~L}$ & -3.6 \\
\hline 42 & $04: 27: 58.5$ & $+18: 45: 30$ & 0.020 & 0.008 & -1.00 & $*$ & HG 7-224 & $\operatorname{lm}$ & V14.3 & & 6.8 & $\mathrm{~L}$ & -1.6 \\
\hline 44 & $04: 27: 59.4$ & $+18: 29: 50$ & 0.027 & 0.009 & -0.15 & -1.00 & $\mathrm{BD}+18639$ & $\mathrm{~K} 2$ & V10.1 & & 15.8 & $\mathrm{~L}$ & -3.2 \\
\hline 43 & 04:29:00.8 & $+18: 40: 01$ & 0.032 & 0.010 & 0.58 & 0.14 & HG 7-237 & $\operatorname{lm}$ & V13.2 & & 25.3 & $\mathrm{~L}$ & -1.8 \\
\hline 48 & 04:30:04.3 & $+18: 13: 42$ & 0.113 & 0.018 & 0.96 & 0.13 & HD $285846^{\ddagger}$ & G5V:e & V10.6 & & 9.4 & $\mathrm{~L}$ & -2.3 \\
\hline 82 & 04:33:24.1 & $-14: 22: 54$ & 0.027 & 0.013 & 1.00 & -0.18 & & & $\mathrm{~J} 15.79$ & & 6.5 & $\mathrm{~L}$ & -0.9 \\
\hline 81 & $04: 34: 26.5$ & $-13: 34: 18$ & 0.043 & 0.016 & 1.00 & 0.45 & 1E0432.1-1341 & GV & V 9 & & 5.5 & $\mathrm{~L}$ & -3.4 \\
\hline 83 & 04:35:19.7 & $-14: 54: 47$ & 0.089 & 0.022 & 0.05 & 0.35 & $(*)$ & $\mathrm{K} 6-7$ & $\mathrm{~J} 14.75$ & & 13.2 & $\mathrm{~L}$ & -0.8 \\
\hline 80 & 05:20:30.0 & $-05: 48: 18$ & 0.037 & 0.012 & 1.00 & -0.01 & $\mathrm{EM}^{*}$ StHA 39 & & V13.5 & & 15.0 & $\mathrm{~L}$ & -1.7 \\
\hline 8 & 09:29:49.2 & $+65: 22: 53$ & 0.014 & 0.006 & 0.48 & -1.00 & RadioS (*) & & 16.19 & 1.55 & 0.0 & LX & -1.0 \\
\hline 5 & 09:32:15.9 & $+70: 06: 35$ & 0.010 & 0.006 & 1.00 & 1.00 & & & 20.10 & $<0.10$ & 27.9 & $\mathrm{LX}$ & 0.4 \\
\hline 6 & 09:34:17.4 & $+66: 21: 50$ & 0.020 & 0.009 & -0.38 & $*$ & & K0III & 13.07 & 1.79 & 30.7 & LX & -2.1 \\
\hline 7 & $09: 37: 27.2$ & $+65: 38: 28$ & 0.047 & 0.011 & 1.00 & -0.08 & $z=0.29, \mathrm{RS}\left(^{*}\right)$ & AGN & 16.52 & 0.98 & 5.4 & $\mathrm{LX}$ & -0.3 \\
\hline 90 & $13: 58: 17.2$ & $-39: 45: 38$ & 0.019 & 0.012 & $*$ & $*$ & & & J16.75 & & 7.6 & & -0.7 \\
\hline 87 & 15:19:18.4 & $-29: 43: 18$ & 0.045 & 0.013 & 0.90 & 0.60 & $(*)$ & M0V & $\mathrm{J} 13.83$ & & 6.0 & LX & -1.4 \\
\hline 84 & 15:37:06.5 & $-24: 47: 35$ & 0.041 & 0.011 & 1.00 & 0.77 & & & J16.78 & & 5.8 & LX & -0.3 \\
\hline 86 & 15:41:07.1 & $-26: 56: 32$ & 0.035 & 0.013 & 0.45 & -0.09 & $(*)$ & K3 & $\mathrm{J} 12.18$ & & 6.7 & LX & -2.2 \\
\hline 85 & $15: 42: 08.5$ & $-26: 29: 17$ & 0.028 & 0.010 & 0.99 & -0.13 & & & $\mathrm{~J} 17.26$ & & 17.3 & $\mathrm{LX}$ & -0.3 \\
\hline 78 & 15:51:00.6 & $-01: 41: 31$ & 0.019 & 0.008 & 1.00 & -0.28 & & & $\mathrm{~J} 21.68$ & & & LX & 1.3 \\
\hline 79 & $15: 53: 22.3$ & $-03: 01: 51$ & 0.127 & 0.019 & -0.08 & 0.07 & SAO 140822 & F5 & V 7.4 & & 16.0 & LX & -3.6 \\
\hline 16 & $16: 44: 19.3$ & $+59: 57: 43$ & 0.022 & 0.005 & 0.18 & 0.59 & SAO 17203 & G5 & V 8.1 & & 12.9 & $\mathrm{LX}$ & -4.0 \\
\hline 15 & $16: 44: 35.2$ & $+59: 58: 43$ & 0.011 & 0.003 & 1.00 & 0.24 & & & 17.89 & 4.25 & 8.0 & LX & -0.4 \\
\hline 17 & $16: 45: 47.8$ & $+59: 57: 36$ & 0.019 & 0.004 & 1.00 & 0.09 & $z=0.23\left(^{*}\right)$ & AGN & 17.31 & 0.70 & 13.1 & LX & -1.1 \\
\hline 13 & $16: 55: 33.5$ & $+61: 39: 47$ & 0.006 & 0.002 & 1.00 & 0.10 & & & 15.68 & 1.77 & 24.2 & LX & -1.6 \\
\hline
\end{tabular}


Table 2. continued

\begin{tabular}{|c|c|c|c|c|c|c|c|c|c|c|c|c|c|}
\hline \multirow[b]{2}{*}{ ID } & \multicolumn{6}{|c|}{ X-ray Source Parameters } & \multicolumn{2}{|c|}{ Identification } & \multicolumn{3}{|c|}{ POSS } & \\
\hline & $\alpha_{(2000)}$ & $\delta_{(2000)}$ & Rate & Error & HR1 & HR2 & Name & Type & $m$ & $\mathrm{O}-\mathrm{E}$ & dist & $\mathrm{B}$ & $\log$ \\
\hline 14 & $16: 58: 14.4$ & $+60: 56: 15$ & 0.011 & 0.004 & -0.25 & 0.28 & & & 16.44 & 3.01 & 14.6 & LX & -1.0 \\
\hline 12 & $16: 58: 32.4$ & $+61: 59: 46$ & 0.007 & 0.003 & 0.76 & 0.07 & RadioS $(*)$ & & 14.64 & 2.44 & 22.4 & LX & -1.9 \\
\hline 9 & $16: 59: 59.2$ & $+62: 14: 45$ & 0.009 & 0.003 & 0.37 & 0.51 & & & 16.95 & 1.15 & 8.4 & LX & -0.9 \\
\hline 11 & 17:00:00.1 & $+62: 02: 18$ & 0.014 & 0.003 & 0.92 & 0.18 & & & 15.67 & 0.57 & 9.3 & LX & -1.2 \\
\hline 10 & $17: 01: 12.1$ & $+62: 06: 31$ & 0.043 & 0.006 & 0.12 & 0.28 & $z=0.32(*)$ & AGN & 15.98 & 1.51 & 23.1 & LX & -0.6 \\
\hline 89 & $19: 15: 11.1$ & $-36: 47: 17$ & 0.020 & 0.010 & $*$ & $*$ & & & J14.00 & & 16.0 & LX & -1.7 \\
\hline 88 & $19: 19: 31.3$ & $-36: 39: 25$ & 0.025 & 0.012 & -1.00 & $*$ & SAO 211169 & F7V & V 7.2 & & 5.3 & LX & -4.4 \\
\hline 4 & $19: 23: 26.0$ & $+70: 07: 48$ & 0.022 & 0.005 & -0.22 & 0.22 & $(*)$ & $\mathrm{K} 6-7$ & 7.75 & 3.04 & 21.0 & LX & -4.2 \\
\hline 3 & $19: 24: 05.7$ & $+70: 19: 56$ & 0.008 & 0.003 & 0.91 & -0.18 & $(*)$ & $\mathrm{AGN}$ & 17.68 & 3.98 & 20.5 & LX & -0.7 \\
\hline 2 & $20: 45: 22.1$ & $+81: 04: 37$ & 0.039 & 0.008 & -0.38 & 0.42 & SAO 3462 & G5 & V 9.4 & & 4.3 & LX & -3.3 \\
\hline 1 & $21: 59: 11.8$ & $+82: 26: 22$ & 0.011 & 0.004 & 1.00 & 1.00 & & & 19.19 & 2.23 & 25.4 & LX & 0.1 \\
\hline 26 & $22: 59: 26.9$ & $+24: 40: 54$ & 0.023 & 0.009 & 0.38 & -0.29 & $(*)$ & K3 & 12.79 & 1.88 & 13.7 & LX & -2.2 \\
\hline 25 & 23:00:03.4 & $+24: 44: 51$ & 0.013 & 0.006 & 1.00 & 1.00 & $87 \mathrm{~GB}$ & RadioS & J16.89 & 1.51 & 20.0 & LX & \\
\hline 27 & $23: 02: 10.9$ & $+24: 17: 35$ & 0.012 & 0.006 & 1.00 & -0.08 & $\operatorname{RadioS}(*)$ & RadioS & 17.36 & 0.11 & 18.2 & LX & -0.6 \\
\hline 24 & $23: 02: 47.5$ & $+24: 53: 14$ & 0.018 & 0.008 & -1.00 & $*$ & $(*)$ & M1.5V:e & 17.99 & 2.68 & 8.7 & LX & -0.2 \\
\hline 23 & $23: 03: 56.6$ & $+25: 00: 43$ & 0.017 & 0.007 & 1.00 & 0.41 & & & 17.84 & 1.11 & 12.3 & LX & -0.3 \\
\hline 60 & $23: 04: 26.3$ & $+12: 58: 40$ & 0.015 & 0.007 & 1.00 & 0.00 & & & 18.18 & 0.71 & 7.6 & LX & -0.2 \\
\hline 57 & $23: 04: 51.9$ & $+14: 12: 41$ & 0.014 & 0.007 & 1.00 & 0.14 & & & O21.68 & $<1.68$ & 28.0 & LX & 1.2 \\
\hline 58 & $23: 04: 57.8$ & $+13: 43: 28$ & 0.015 & 0.007 & 1.00 & -0.18 & & & 17.63 & 2.11 & 21.0 & LX & -0.4 \\
\hline 59 & $23: 05: 19.3$ & $+13: 15: 37$ & 0.015 & 0.007 & 1.00 & 0.40 & & & 17.80 & 2.63 & 19.8 & $\mathrm{LX}$ & -0.3 \\
\hline 55 & 23:05:31.1 & $+15: 14: 50$ & 0.011 & 0.006 & 0.53 & 0.61 & $z=0.07(*)$ & $\mathrm{AGN}$ & 18.89 & 1.29 & 6.4 & LX & 0.0 \\
\hline 56 & $23: 05: 58.5$ & $+14: 13: 25$ & 0.017 & 0.007 & 1.00 & -0.09 & Radios $(*)$ & & 16.72 & 1.78 & 14.8 & $\mathrm{LX}$ & -0.7 \\
\hline 38 & $23: 07: 47.0$ & $+19: 02: 46$ & 0.015 & 0.007 & 1.00 & 0.56 & & & 16.23 & 1.54 & 30.5 & $\mathrm{LX}$ & -1.0 \\
\hline 39 & $23: 07: 47.1$ & $+19: 01: 17$ & 0.036 & 0.011 & 1.00 & -0.06 & [HB89] 2305+] & 187 QSO & 15.80 & 1.01 & 21.4 & $\mathrm{~L}$ & -0.8 \\
\hline 35 & $23: 08: 12.3$ & $+19: 30: 09$ & 0.021 & 0.008 & 1.00 & 0.23 & & & 18.34 & 1.11 & 25.7 & $\mathrm{~L}$ & 0.0 \\
\hline 53 & $23: 08: 28.1$ & $+15: 55: 46$ & 0.019 & 0.008 & 0.03 & -0.08 & & & 19.86 & 1.75 & 2.5 & LX & 0.6 \\
\hline 51 & 23:08:41.6 & $+17: 49: 02$ & 0.012 & 0.006 & 1.00 & -1.00 & & & 15.79 & 1.91 & 28.5 & $\mathrm{LX}$ & -1.2 \\
\hline 54 & $23: 08: 42.8$ & $+15: 32: 51$ & 0.045 & 0.012 & 0.21 & -0.64 & HD 218539 & G0 & V 9.7 & & 4.5 & LX & -3.1 \\
\hline 47 & 23:09:01.5 & $+18: 20: 21$ & 0.008 & 0.005 & 1.00 & 1.00 & $\mathrm{BD}+174873$ & G5 & V 9.2 & & & LX & -4.0 \\
\hline 28 & 23:09:01.8 & $+23: 31: 35$ & 0.027 & 0.009 & 1.00 & 0.53 & & & 18.74 & 1.11 & 6.8 & $\mathrm{LX}$ & 0.3 \\
\hline 46 & 23:09:07.4 & $+18: 25: 20$ & 0.030 & 0.011 & -0.03 & 0.24 & HD 218602 & G5 & 16.35 & 1.51 & 14.9 & $\mathrm{X}$ & -0.6 \\
\hline 29 & $23: 09: 44.7$ & $+22: 34: 18$ & 0.019 & 0.007 & 1.00 & 0.47 & & & O21.89 & $<1.89$ & 17.3 & LX & 1.4 \\
\hline 49 & $23: 10: 59.8$ & $+18: 08: 29$ & 0.032 & 0.010 & -1.00 & $*$ & $(*)$ & DC WD & 18.00 & -0.55 & 22.0 & $\mathrm{LX}$ & 0.1 \\
\hline 45 & $23: 11: 02.7$ & $+18: 28: 20$ & 0.012 & 0.006 & 0.98 & 1.00 & & & & & & LX & $>0.8$ \\
\hline 50 & $23: 12: 31.2$ & $+18: 06: 22$ & 0.010 & 0.005 & 1.00 & 1.00 & & & 18.42 & 1.96 & 24.3 & LX & -0.3 \\
\hline 36 & $23: 12: 36.6$ & $+19: 23: 03$ & 0.025 & 0.009 & 0.51 & 0.20 & binary $(*)$ & K3-5 & & & & $\mathrm{LX}$ & \\
\hline 19 & $23: 25: 14.6$ & $+33: 14: 12$ & 0.021 & 0.007 & 0.18 & 0.13 & $(*)$ & K3 & 9.36 & 1.94 & 7.8 & LX & -3.6 \\
\hline 20 & $23: 25: 25.8$ & $+33: 12: 44$ & 0.016 & 0.006 & -1.00 & $*$ & $(*)$ & K3 & 10.78 & 2.43 & 32.5 & $\mathrm{LX}$ & -3.1 \\
\hline 18 & $23: 25: 57.4$ & $+33: 17: 19$ & 0.013 & 0.005 & 1.00 & 0.48 & & & 18.34 & $>2.94$ & 8.9 & LX & -0.2 \\
\hline
\end{tabular}


Table 3. Remarks on the identification of each object. A plus sign indicates a secure identification

\begin{tabular}{|c|c|c|}
\hline$\overline{\overline{\mathrm{ID}}}$ & & Remark \\
\hline$\overline{01}$ & & $\begin{array}{l}\text { no object on POSS, } 3 \text { red objects detected in CCD frames, object B is most prominent } \\
\text { in } I \text { band, tentative identification: B }\end{array}$ \\
\hline 02 & + & bright star \\
\hline 03 & + & faint object shows broad Balmer lines \\
\hline 04 & + & $\begin{array}{l}5 \text { objects visible in error circle, all show late stellar spectra, A is by far the brightest } \\
(\mathrm{K} 6-7)\end{array}$ \\
\hline 05 & & no radio source, no POSS source, faint red object in $30 \mathrm{~s}$ cosmic pointing \\
\hline 06 & + & $\mathrm{K}$ star just on error circle (nothing else in $600 \mathrm{~s} 60$-inch $\mathrm{R}$ ) \\
\hline 07 & + & 2 objects within error circle. Brightest object shows AGN spectrum \\
\hline 08 & + & $\begin{array}{l}\text { radio source right at X-ray position, no POSS object, no object in } 60 \text {-inch frames but } \\
\text { in Cosmic frame }(60 \mathrm{~s}) \text { point like object discovered in } B, V \text { and } R \text { at radio position, } \\
\text { object } \mathrm{A}\end{array}$ \\
\hline 09 & & 1 object, no spectrum \\
\hline 10 & + & 2 objects, brighter is star, fainter shows AGN lines \\
\hline 11 & & 1 extended object $(\mathrm{A})$, no spectrum \\
\hline 12 & + & 2 objects, fainter object $(\mathrm{R})$ coincides with radio source \\
\hline 13 & & 1 bright object $(\mathrm{A})$, no spectrum, $\mathrm{B}$ is reddest object \\
\hline 14 & & 1 brighter object $(\mathrm{A})$, no spectrum \\
\hline 15 & & only $\mathrm{A}$ is detected in $B$ image, in $R$ and $I$ a faint object is visible next to A \\
\hline 16 & + & bright SAO star \\
\hline 17 & + & 1 object, AGN spectrum \\
\hline 18 & & $\mathrm{~A}$ and $\mathrm{B}$ are detected in $R$ and $I$ frame, $\mathrm{A}$ is brightest in $\mathrm{I}$ \\
\hline 19 & + & bright star, K3 \\
\hline 20 & + & brightest star of 6 objects is K star, apparently extended object is a blend of 2 \\
\hline 21 & & A deep in emission cloud, pre-main sequence object? \\
\hline 22 & + & bright $\mathrm{K} 0$ star $\mathrm{BD}+30547$ \\
\hline 23 & & 2 objects, no spectra \\
\hline 24 & + & brightest object is $\mathrm{M}$ dwarf with emission lines \\
\hline 25 & + & $\begin{array}{l}\text { A is K0III star, position of B is compatible with radio source (faint and red), X-ray } \\
\text { source is hard, so identify B as counterpart }\end{array}$ \\
\hline 26 & + & $\begin{array}{l}1 \text { bright star inside error circle (B) a brighter star north-west from that (A) both are } \\
\text { K3 stars (pick B as counterpart) }\end{array}$ \\
\hline 27 & + & $\begin{array}{l}\text { radio source coincides with } R \text {. A is a nebula that shows no significant line emission } \\
\left(\text { no } \mathrm{H}_{\alpha} \text { no }[\mathrm{OIII}]\right)\end{array}$ \\
\hline 28 & & A is detected in $B, R$ and $I$ frames \\
\hline 29 & & bright star south-east of X-ray position $(\mathrm{S})$ is likely counterpart \\
\hline 30 & + & $\begin{array}{l}2 \text { objects, brighter on is late type star, fainter one coincides with radio source, shows } \\
\text { AGN lines, B is counterpart }\end{array}$ \\
\hline 31 & + & $\begin{array}{l}\text { MS Source, type QSO, not rediscovered in radio pointing, only southern part of close } \\
\text { pair (A) is detected in B }\end{array}$ \\
\hline 32 & + & bright star \\
\hline 33 & + & $\begin{array}{l}2 \text { objects both diametrically opposite on the error circle, B is late type star, A is } \\
\text { M-dwarf with strong H-alpha emission }\end{array}$ \\
\hline 34 & + & T-Tauri \\
\hline 35 & & several objects, $\mathrm{C}$ is reddest \\
\hline 36 & + & K star binary not resolved on POSS \\
\hline 37 & + & Einstein source, bright M-star \\
\hline 38 & & no data \\
\hline 39 & + & Quasar HB89 \\
\hline 40 & + & A coincides with radio source \\
\hline
\end{tabular}


Table 3. continued

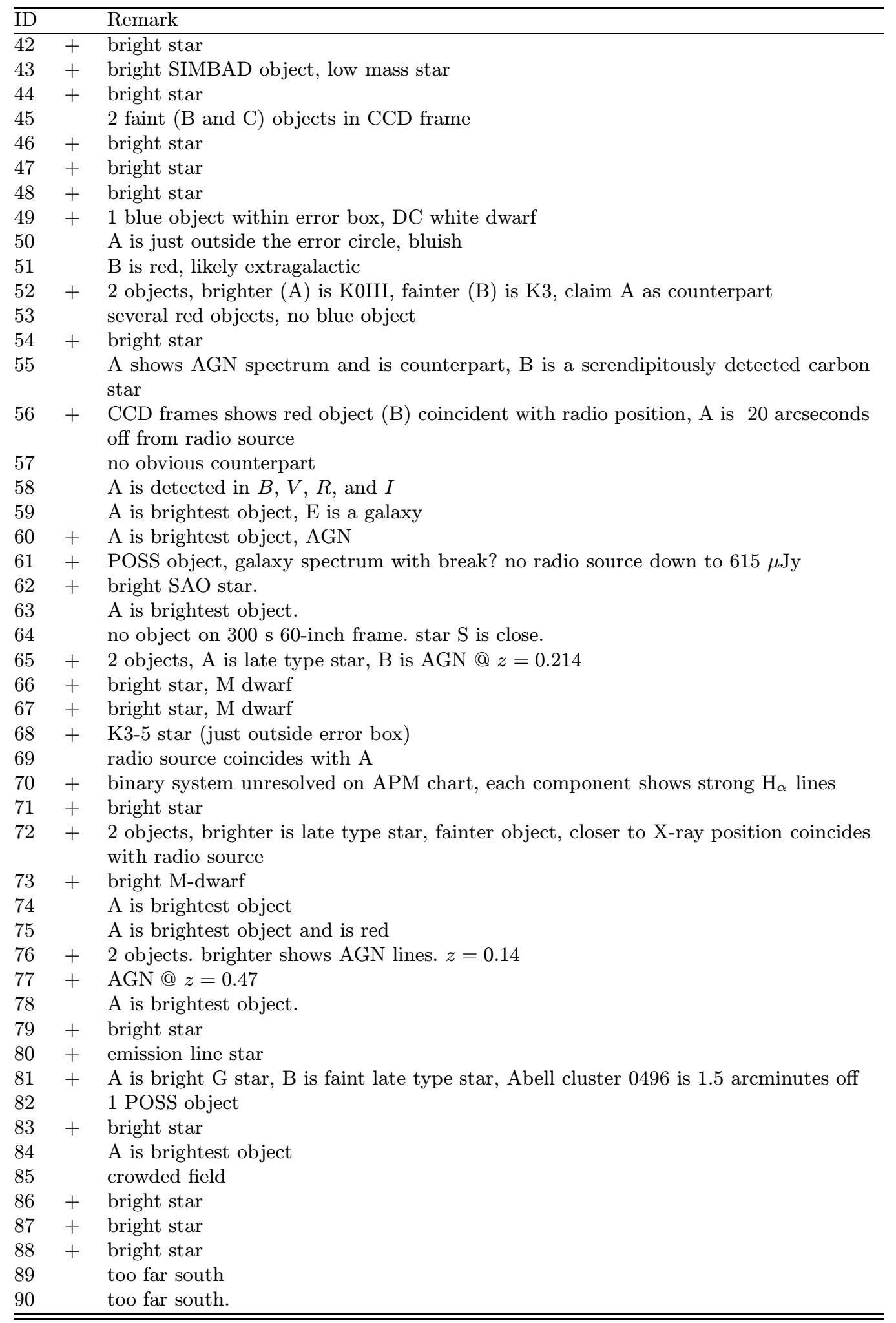



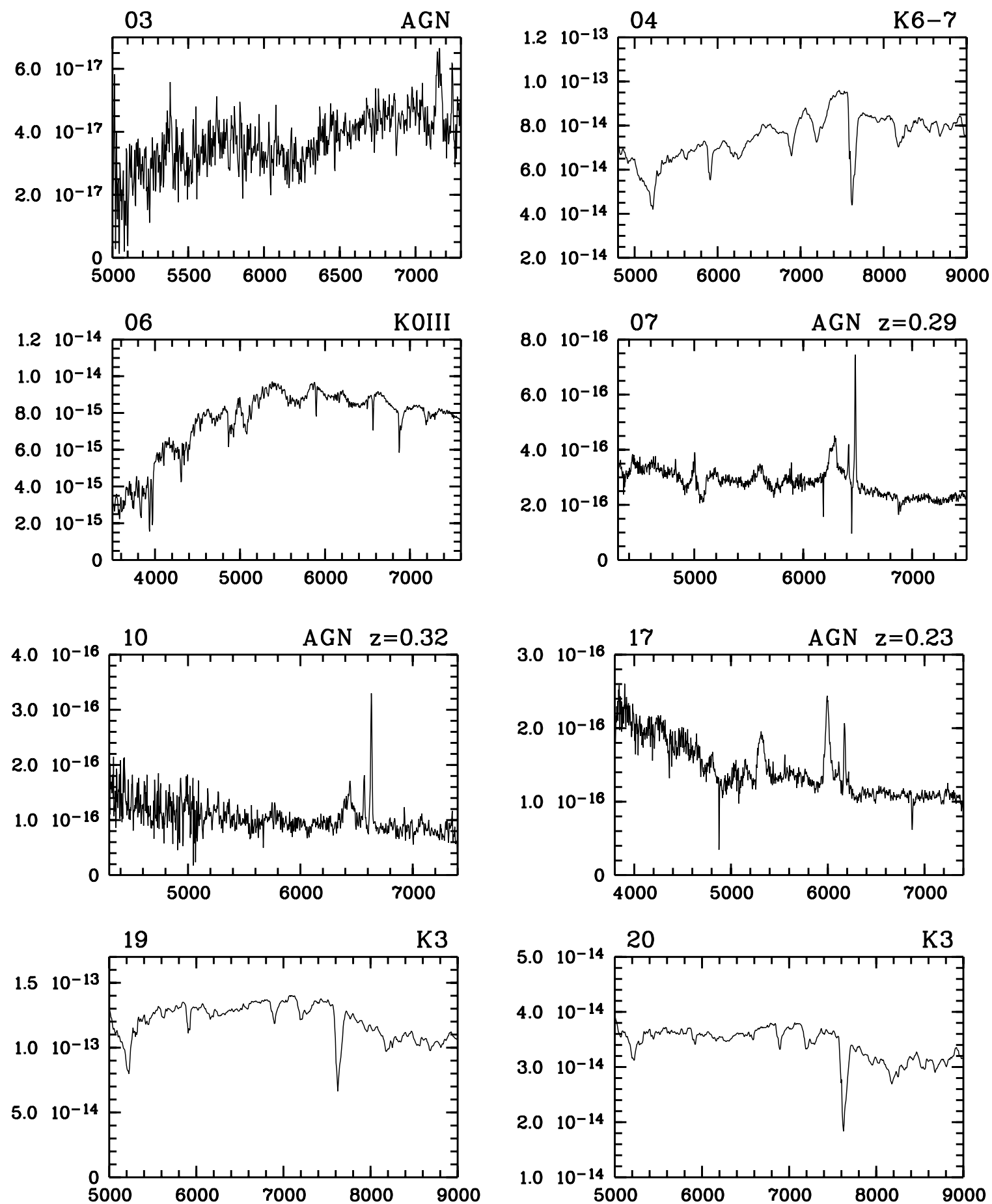

Fig. 8. Optical spectra. The internal ID and the spectral classification are given on top of each spectrum. The $x$-axis shows the wavelength in Angstrom and the $y$-axis the flux in arbitrary units. Remarks: 07: AGN, $z=0.29$, [NeIII], H ${ }_{\beta}$, [OIII] 4959 $\AA$, 5007 Å. 10: AGN, $z=0.32, \mathrm{H}_{\beta}$, [OIII] $4959 \AA$, $5007 \AA$; 17: AGN, $z=0.22, \mathrm{H}_{\gamma}, \mathrm{H}_{\beta}$, [OIII] $4959 \AA$, $5007 \AA$ 

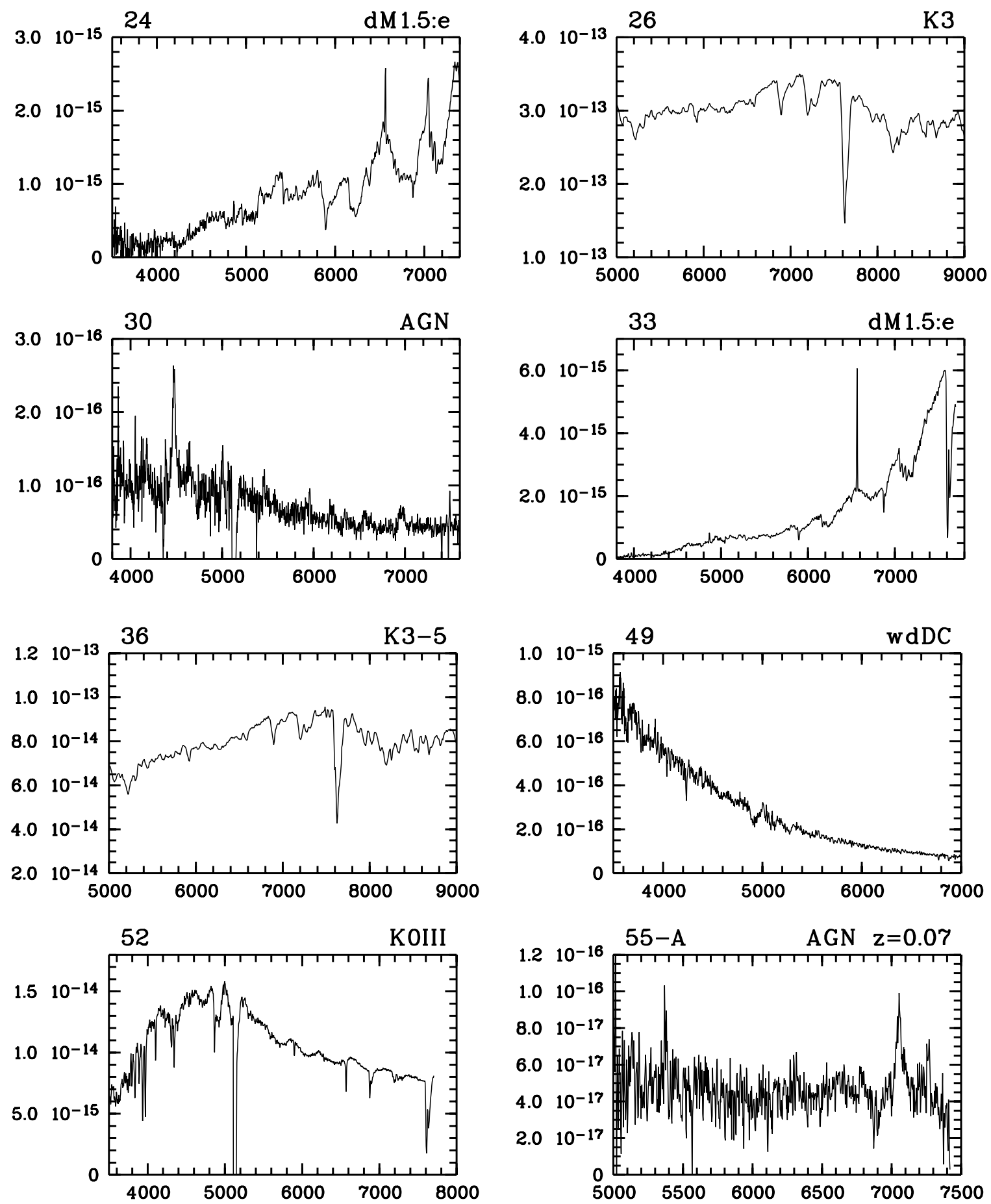

Fig. 8. continued. Remarks: 24: dM1.5:e, $\mathrm{H}_{\beta}$ and $\mathrm{H}_{\alpha}$ in emission; 33: dM1.5:e, strong $\mathrm{H}_{\alpha}$ emission, some $\mathrm{H}_{\beta}$. 49: DC white dwarf, the feature close to $5000 \AA$ is probably due to a mismatch between the red and the blue side of the spectrograph; 52: K0III, feature is due to red/blue mismatch; 55-A: AGN, $z=0.074$, [0III] $5007 \AA$, HeI, $\mathrm{H}_{\alpha}$, [SII] $6731 \AA$ 

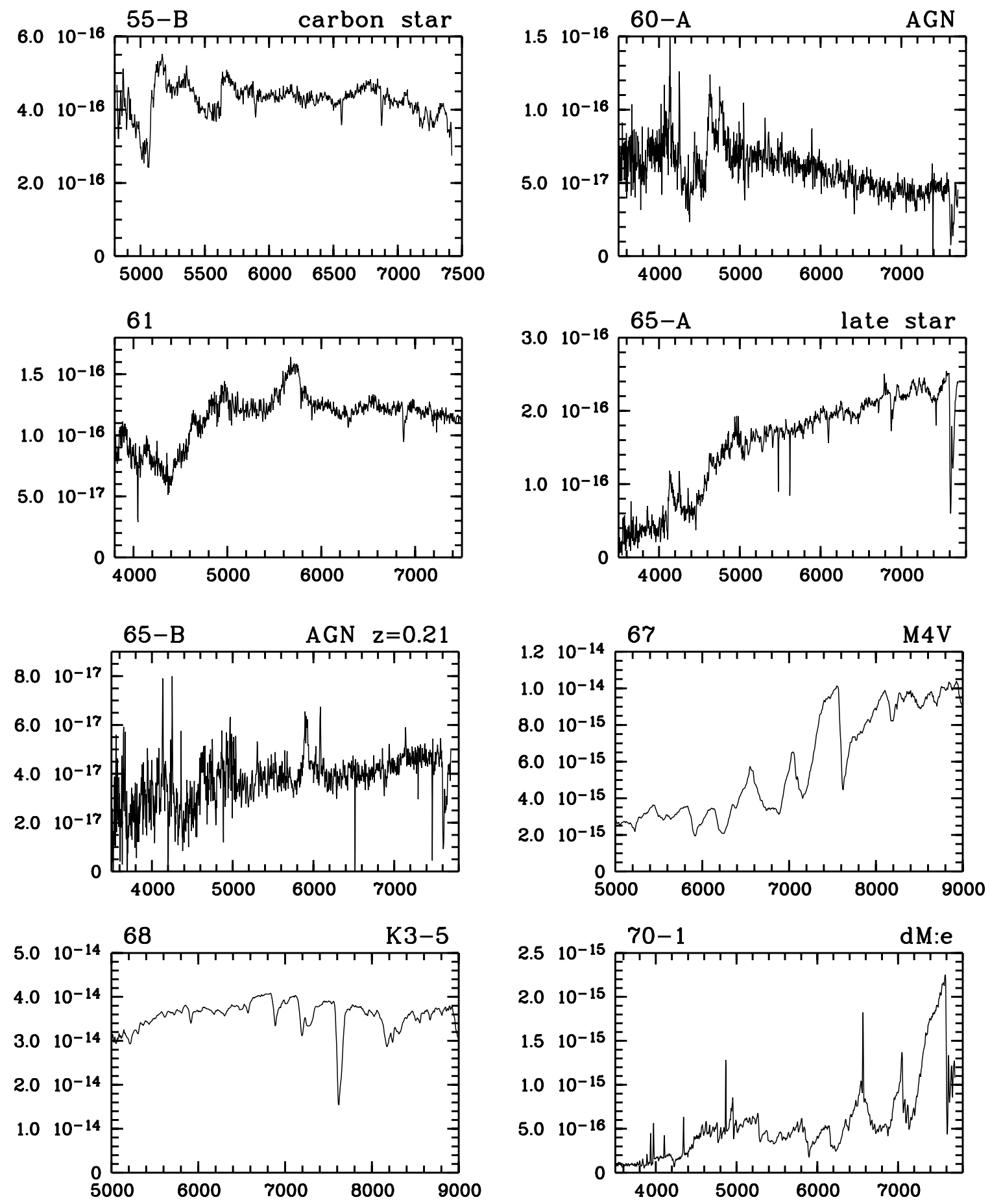

Fig. 8. continued. Remarks: 70-1: binary system of two emission line M-dwarfs 

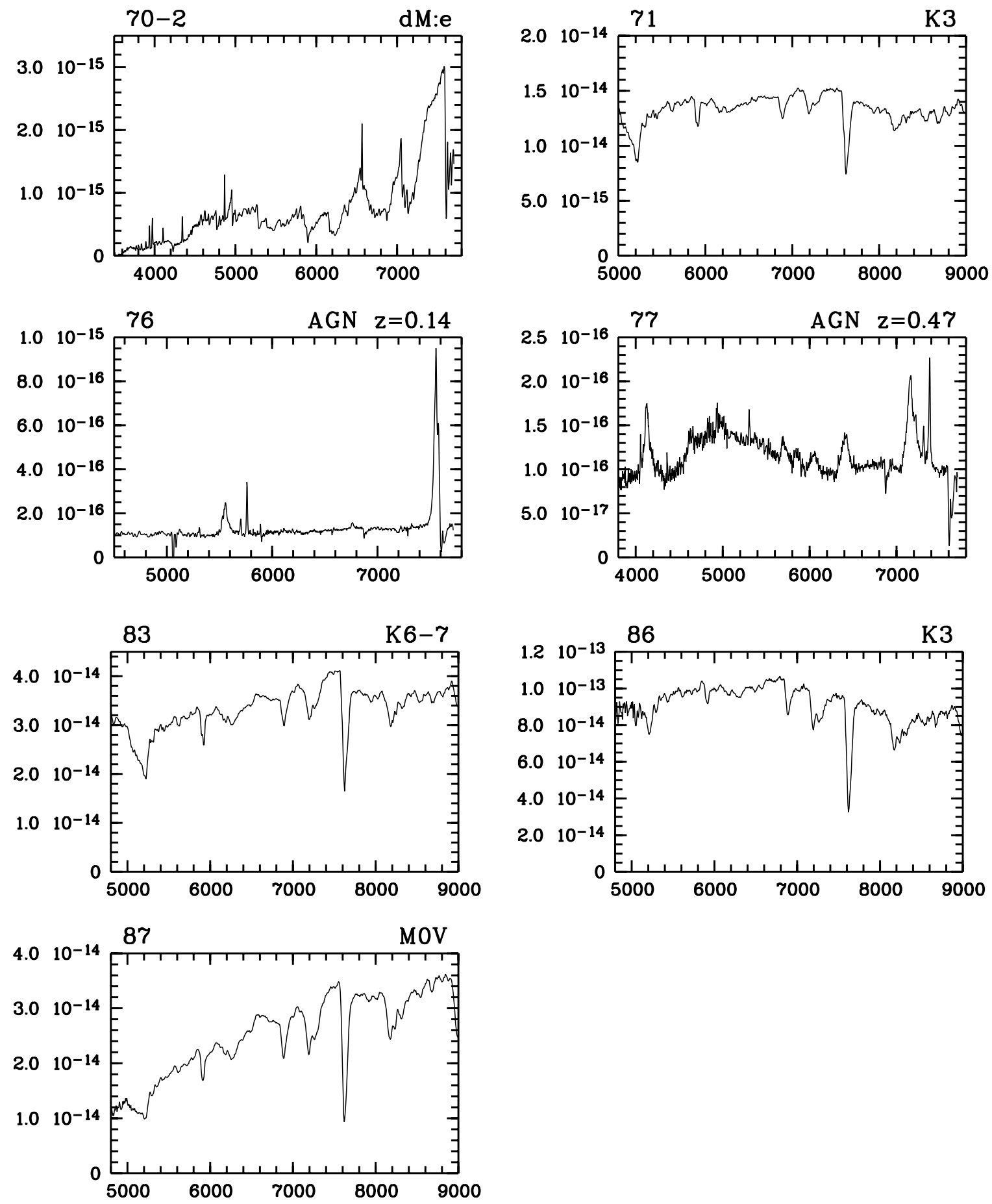

Fig. 8. continued. Remarks: 70-2: second component of 70-1; 76: AGN, $z=0.14, \mathrm{H}_{\beta}$, [OIII] $4959 \AA$ A, $5007 \AA$, $\mathrm{H}_{\alpha} ; \mathbf{7 7}: \mathrm{AGN}$, $z=0.47, \mathrm{H}_{\delta}, \mathrm{H}_{\gamma}$, [OIII] $4363 \AA, \mathrm{H}_{\beta}$, [OIII] $4959 \AA, 5007 \AA$ 